\title{
On Solutions of the Fuji-Suzuki-Tsuda System
}

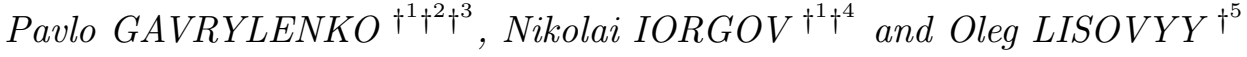 \\ $\dagger^{1}$ Bogolyubov Institute for Theoretical Physics, 03143 Kyiv, Ukraine \\ E-mail: pasha145@gmail.com,iorgov@bitp.kiev.ua \\ $\dagger^{2}$ Center for Advanced Studies, Skolkovo Institute of Science and Technology, \\ 143026 Moscow, Russia \\ $\dagger^{3}$ National Research University Higher School of Economics, International Laboratory \\ of Representation Theory and Mathematical Physics, Moscow, Russia \\ $\dagger^{4}$ Kyiv Academic University, 36 Vernadsky Ave., 03142 Kyiv, Ukraine \\ $\dagger^{5}$ Institut Denis-Poisson, Université de Tours, Parc de Grandmont, 37200 Tours, France \\ E-mail: lisovyi@lmpt.univ-tours.fr
}

Received June 22, 2018, in final form October 30, 2018; Published online November 11, 2018

https://doi.org/10.3842/SIGMA.2018.123

\begin{abstract}
We derive Fredholm determinant and series representation of the tau function of the Fuji-Suzuki-Tsuda system and its multivariate extension, thereby generalizing to higher rank the results obtained for Painlevé VI and the Garnier system. A special case of our construction gives a higher rank analog of the continuous hypergeometric kernel of Borodin and Olshanski. We also initiate the study of algebraic braid group dynamics of semi-degenerate monodromy, and obtain as a byproduct a direct isomonodromic proof of the AGT-W relation for $c=N-1$.
\end{abstract}

Key words: isomonodromic deformations; Painlevé equations; Fredholm determinants

2010 Mathematics Subject Classification: 33E17; 34M55; 34M56

\section{Introduction}

The purpose of this note is to initiate a systematic study of rank $N$ Fuji-Suzuki-Tsuda system, abbreviated below as $\mathrm{FST}_{N}$. This Hamiltonian system of nonlinear non-autonomous ODEs first appeared as a particular reduction of the Drinfeld-Sokolov hierarchy [12, 28], and independently in [29] as a reduction of the universal character hierarchy. Its fundamental significance comes from the isomonodromic theory [11], where it describes deformations of rank $N$ Fuchsian systems with 4 regular singular points, 2 of which have special spectral type $(N-1,1)[29,31]$. Following [14], we refer to such linear systems as semi-degenerate.

For $N=2$, the spectral profile remains unconstrained and the corresponding $\mathrm{FST}_{2}$ system is equivalent to the sixth Painlevé equation (PVI). For general $N$, the dimension of the phase space of $\mathrm{FST}_{N}$ is $2(N-1)$, which is to be compared with the dimension $2(N-1)^{2}$ of the system of deformation equations for generic 4-point Fuchsian case. The $\mathrm{FST}_{N}$ system is thus the closest relative of PVI in higher rank. It generalizes PVI in the direction different from the much studied Garnier system, which corresponds to increasing the number of singular points while keeping fixed the rank $N=2$ of the associated linear problem. Loosely speaking, going from PVI to $\mathrm{FST}_{N}$ is a nonlinear counterpart of the generalization of the Gauss ${ }_{2} F_{1}$ to ClausenThomae ${ }_{N} F_{N-1}$ hypergeometric function; the Garnier system would correspond to multivariate

This paper is a contribution to the Special Issue on Painlevé Equations and Applications in Memory of Andrei Kapaev. The full collection is available at https://www.emis.de/journals/SIGMA/Kapaev.html 
functions of Lauricella type. This analogy is exhibited already at the level of special function solutions but in fact it goes much further: we will see that the general Fredholm determinant solution of $\mathrm{FST}_{N}$ can be constructed from the fundamental solutions of two auxiliary linear

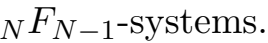

Besides serving as a model example for isomonodromic deformations in higher rank, the $\mathrm{FST}_{N}$ system appears in a number of applications. Its tau function is given by a Fourier transform of 4point semi-degenerate conformal blocks of the Toda CFT with central charge $c=N-1$ [14]. By the AGT-W correspondence [1, 10, 24, 32], it thus coincides with the dual instanton partition of $\mathcal{N}=2 \mathrm{U}(N)$ gauge theory with $N$ fundamental and $N$ anti-fundamental matter hypermultiplets in the self-dual $\Omega$-background. Quantized $\mathrm{FST}_{N}$ system also appears in this context [33].

On the other hand, little is known about the actual solutions of $\mathrm{FST}_{N}$. The present paper begins to explore an agenda of related issues, to a large extent determined by the Painlevé VI state-of-the-art. Our main tool is the Riemann-Hilbert correspondence which translates the questions on solutions of the nonlinear isomonodromic system in terms of monodromy of the associated linear problem. For example, all known PVI solutions expressible in terms of elementary or classical special functions can be divided into 3 (overlapping) categories:

- Riccati solutions. They correspond to monodromy representations generated from the reducible ones by Painlevé VI affine symmetry transformations [26]. The Riccati tau functions are expressed as finite determinants with hypergeometric ${ }_{2} F_{1}$-entries. Their $\mathrm{FST}_{N}$ analogs were investigated in $[22,27,30]$ and similarly involve ${ }_{N} F_{N-1}$.

- Picard solutions. These solutions $[7,19,23]$ are expressed in terms of elliptic functions, with the relevant monodromy matrices given by quasi-permutations. Their higher rank generalization was introduced in [20] without imposing an extra condition of semi-degenerate monodromy. There is, however, a simple instance of quasi-permutation monodromy given by permutation of only two basis vectors. Its spectrum $\{1, \ldots, 1,-1\}$ satisfies the semidegeneracy condition. An interesting class of algebro-geometric $\mathrm{FST}_{N}$ solutions is thus associated to monodromy corresponding to 2 elementary permutations and 2 arbitrary quasi-permutations.

- Algebraic solutions. This class corresponds to finite orbits of an action of the pure braid group $\mathcal{P}_{3}$ on the PVI monodromy manifold [9]. The classification of such orbits was accomplished in [21]. In Section 3 of the present work, we describe the algebraic braid group dynamics on $\mathrm{FST}_{N}$ monodromy data and set up a classification problem to be solved.

The solution of Painlevé VI corresponding to monodromy in general position was expressed as an explicit Fredholm determinant in [15]; see [5] for substantially simplified proof. The relevant integral operator acts on $\mathbb{C}^{2} \otimes L^{2}\left(S^{1}\right)$ and its integrable kernel is written in terms of solutions of two hypergeometric systems. The determinant expansion further yields a series representations for the PVI tau function. The present work extends both determinant and series representations to the case of the $\mathrm{FST}_{N}$ system (Sections 4 and 5).

When one of the auxiliary systems has reducible monodromy and one of the relevant monodromy matrices generates a nilpotent subgroup of $\mathrm{SL}(2, \mathbb{C})$, the block integral kernel of $[15$, Theorem A] can be reduced to a scalar continuous hypergeometric kernel on an interval. The corresponding PVI solution first appeared in [3]; it generalizes the well-known sine- and Airykernel Painlevé V and II transcendents. We obtain an $\mathrm{FST}_{N}$ analog of this solution in Section 6. It would be interesting to understand whether, similarly to the $N=2$ case [4], it plays a role in the harmonic analysis on infinite groups such as $\mathrm{U}(\infty)$.

The last section of this manuscript is devoted to a multivariate generalization of the $\mathrm{FST}_{N}$ system, denoted by $\mathcal{G}_{N, n-3}$ in [31]. The corresponding Fuchsian system has $n$ regular singular 
points, of which all but 2 have semi-degenerate spectral type $(N-1,1)$. We present the Fredholm determinant and series representation of the corresponding tau function and identify the coefficients of the latter with Nekrasov functions [25] for $\mathcal{N}=2 \mathrm{U}(N)$ linear quiver gauge theory. In combination with the results of [14], this gives a direct proof of the AGT-W correspondence for $c=N-1$ (Appendix A).

\section{Semi-degenerate Fuchsian system and FST $_{N}$}

This section explains the relation of $\mathrm{FST}_{N}$ system and monodromy preserving deformations. The relevant results are essentially extracted from [31].

Let $G=\operatorname{GL}(N, \mathbb{C})$. Consider a Fuchsian system with 4 regular singular points on $\mathbb{C P}^{1}$,

$$
\partial_{z} \Phi=\Phi A(z), \quad A(z)=\sum_{\nu=0,1, t} \frac{A_{\nu}}{z-\nu}, \quad \text { with } \quad A_{0,1, t} \in \operatorname{Mat}_{N \times N}(\mathbb{C}) .
$$

Denote $A_{\infty}:=-\sum_{\nu=0,1, t} A_{\nu}$. Any locally defined fundamental matrix solution $\Phi(z)$ can be analytically continued to any simply connected domain in $\mathbb{C} \backslash\{0,1, t\}$. Its global analytic properties are encoded in the monodromy representation $m \in \operatorname{Hom}\left(\pi_{1}(\mathbb{C} \backslash\{0,1, t\}), G\right)$ associated to (2.1). Choosing the generators $\gamma_{0,1, t, \infty}$ of the fundamental group as shown in Fig. 1, the monodromy of $\Phi(z)$ is described by their 4 images $M_{\nu}=m\left(\gamma_{\nu}\right)$ which satisfy $M_{0} M_{t} M_{1} M_{\infty}=\mathbb{1}$. The freedom to choose an arbitrary basis of solutions means that the linear system (2.1) only defines the conjugacy class of monodromy.

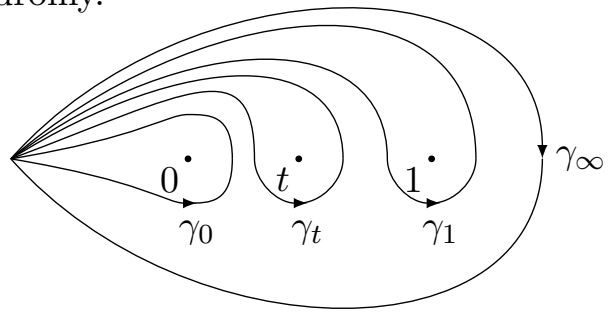

Figure 1. Generators $\left\{\gamma_{\nu}\right\}$.

If the eigenvalues of each $A_{\nu}(\nu=0,1, t, \infty)$ do not differ by non-zero integers, the monodromy preserving deformation of (2.1) is described by the equations

$$
\begin{aligned}
& \frac{\mathrm{d} A_{0}}{\mathrm{~d} t}=\frac{\left[A_{0}, A_{t}\right]}{t}+\left[A_{0}, K\right], \quad \frac{\mathrm{d} A_{1}}{\mathrm{~d} t}=\frac{\left[A_{1}, A_{t}\right]}{t-1}+\left[A_{1}, K\right], \\
& \frac{\mathrm{d} A_{t}}{\mathrm{~d} t}=-\frac{\left[A_{0}, A_{t}\right]}{t}-\frac{\left[A_{1}, A_{t}\right]}{t-1}+\left[A_{t}, K\right], \quad K=L^{-1} \frac{\mathrm{d} L}{\mathrm{~d} t} .
\end{aligned}
$$

Here $L(t) \in \mathrm{GL}(N, \mathbb{C})$ is an arbitrary invertible matrix function of $t$ corresponding to the choice of the gauge of $\Phi(z)$ at $z=\infty$. It can be absorbed by setting $A_{\nu}=L^{-1} \tilde{A}_{\nu} L$. Although one may be tempted to set $L(t)=$ const, $K=0$ from the very beginning, below we will also use a more subtle time-dependent gauge. The deformation equations (2.2) appear as the compatibility conditions of the Fuchsian system with

$$
\partial_{t} \Phi=\Phi B(z), \quad B(z)=K-\frac{A_{t}}{z-t} .
$$

The spectra $\operatorname{Spec}\left(A_{\nu}\right)$ give exponents of local monodromy around $z=\nu$ and are therefore conserved under isomonodromic evolution. The main object of interest for us will be the JimboMiwa-Ueno tau function [18] defined by

$$
\frac{\mathrm{d}}{\mathrm{d} t} \ln \tau_{\mathrm{JMU}}(t):=\frac{1}{2} \operatorname{Res}_{z=t} \operatorname{Tr} A^{2}(z)=\frac{\operatorname{Tr} A_{0} A_{t}}{t}+\frac{\operatorname{Tr} A_{1} A_{t}}{t-1} .
$$

The tau function is clearly $L$-independent. 
We are now going to parameterize the matrices $A_{\nu}$ and rewrite the deformation equations $(2.2)$ more explicitly in the semi-degenerate case. Let the singular points $z=1, t$ be of spectral type $(N-1,1)$. Employing if necessary a scalar gauge transformation of the form $\Phi(z) \mapsto$ $(z-t)^{a}(z-1)^{b} \tilde{\Phi}(z)$, it can be assumed that the eigenvalue of $A_{t}$ and $A_{1}$ with multiplicity $N-1$ is equal to 0 ; the non-degenerate eigenvalues will be denoted by $\Lambda_{t}$ and $\Lambda_{1}$. Assume in addition that $A_{0}$ and $A_{\infty}$ remain sufficiently generic so that they can be brought to a lower and upper triangular form:

$$
A_{0}=\left(\begin{array}{cccc}
\theta_{0}^{(1)} & 0 & \ldots & 0 \\
* & \theta_{0}^{(2)} & \ldots & . \\
\vdots & \vdots & \ddots & 0 \\
* & \cdot & * & \theta_{0}^{(N)}
\end{array}\right), \quad A_{\infty}=\left(\begin{array}{cccc}
\theta_{\infty}^{(1)} & * & \cdots & * \\
0 & \theta_{\infty}^{(2)} & \ldots & \cdot \\
\vdots & \vdots & \ddots & * \\
0 & \cdot & 0 & \theta_{\infty}^{(N)}
\end{array}\right)
$$

Write $A_{t, 1}$ in this basis as $A_{t}=-q^{T} \otimes p, A_{1}=-\bar{q}^{T} \otimes \bar{p}$. Here $q, \bar{q}, p, \bar{p}$ are row vectors. The notation for their individual entries is fixed so that, e.g., $q=\left(q^{(1)} \ldots q^{(N)}\right)$. We have the constraints

$$
\Lambda_{t}=-\sum_{k=1}^{N} p^{(k)} q^{(k)}, \quad \Lambda_{1}=-\sum_{k=1}^{N} \bar{p}^{(k)} \bar{q}^{(k)}, \quad \Lambda_{t}+\Lambda_{1}+\sum_{k=1}^{N}\left(\theta_{0}^{(k)}+\theta_{\infty}^{(k)}\right)=0 .
$$

The former two conditions come from the computation of $\operatorname{Tr} A_{t, 1}$ and the $3 \mathrm{rd}$ is the Fuchs relation. The triangular form of $A_{0, \infty}$ and the relation $\sum_{\nu=0,1, t, \infty} A_{\nu}=0$ imply that

$$
\begin{aligned}
& \left(A_{0}\right)_{l k}=p^{(k)} q^{(l)}+\bar{p}^{(k)} \bar{q}^{(l)}, \quad k<l, \\
& \left(A_{\infty}\right)_{l k}=p^{(k)} q^{(l)}+\bar{p}^{(k)} \bar{q}^{(l)}, \quad k>l .
\end{aligned}
$$

If all $\bar{q}^{(k)} \neq 0$, the remaining freedom of diagonal gauge transformations can be used to set $\bar{q}^{(k)}=1$. Considering the diagonal of the same matrix relation $\sum_{\nu=0,1, t, \infty} A_{\nu}=0$, we then obtain

$$
\bar{p}^{(k)}=-p^{(k)} q^{(k)}+\theta_{\infty}^{(k)}+\theta_{0}^{(k)}, \quad k=1, \ldots, N .
$$

We can further set $q^{(1)}=1$ and express $p^{(1)}$ using the 1st of relations (2.4), so that $A(z)$ is completely parameterized by $2(N-1)$ quantities $p^{(k)}, q^{(k)}$ with $k=2, \ldots, N$.

To rewrite the equations (2.2) in these coordinates, we also need the expression for $K$. Note that since $A_{\infty}$ is upper triangular, so are $H$ and $K$. On the other hand, the matrix $B(z=0)=K+\frac{A_{t}}{t}=G_{0}^{-1} \partial_{t} G_{0}$, where $G_{0}$ characterizes the gauge at $z=0$ : namely, $A_{0}=G_{0}^{-1} \operatorname{diag}\left\{\theta_{0}^{(1)}, \ldots, \theta_{0}^{(N)}\right\} G_{0}$. Therefore, $B(z=0)$ is lower triangular, which immediately yields the off-diagonal elements of $K$ :

$$
K_{k l}= \begin{cases}\frac{q^{(k)} p^{(l)}}{t}, & k<l \\ 0, & k>l .\end{cases}
$$

In order to determine the diagonal, one can similarly use that $B(z=1)=K+\frac{A_{t}}{t-1}=G_{1}^{-1} \partial_{t} G_{1}$, where $A_{1}=G_{1}^{-1} \operatorname{diag}\left\{\Lambda_{1}, 0, \ldots, 0\right\} G_{1}$. In the gauge where all $\bar{q}^{(k)}=1$, we can set $\left(G_{1}^{-1}\right)_{k 1}=1$ for $k=1, \ldots, N$, so that $\sum_{k=1}^{N}\left(K+\frac{A_{t}}{t-1}\right)_{l k}=0$. It follows that

$$
K_{l l}=-\frac{q^{(l)}}{t} \sum_{k=l+1}^{N} p^{(k)}+\frac{q^{(l)}}{t-1} \sum_{k=1}^{N} p^{(k)}, \quad l=1, \ldots, N .
$$


The deformation equations (2.2) can now be rewritten as follows. For $k=2, \ldots, N-2$, we have

$$
\begin{aligned}
\frac{\mathrm{d} q^{(k)}}{\mathrm{d} t} & =\sum_{l=1}^{N}\left(f \delta_{k l}-g_{k l}\right) q^{(l)}, \\
\frac{\mathrm{d} p^{(k)}}{\mathrm{d} t} & =\sum_{l=1}^{N} p^{(l)}\left(g_{l k}-f \delta_{k l}\right),
\end{aligned}
$$

where $g=\frac{A_{0}}{t}+\frac{A_{1}}{t-1}+K$ and

$$
f=\sum_{m=1}^{N} g_{1 m} q^{(m)}=\frac{\theta_{0}^{(1)}-\Lambda_{t}}{t}+\frac{\sum_{m=1}^{N} p^{(m)}}{t(t-1)}-\frac{\sum_{m=1}^{N} \bar{p}^{(m)} q^{(m)}}{t-1} .
$$

For $k=1$, the equation (2.5) is satisfied automatically (recall that $q^{(1)}=1$ and $p^{(1)}=-\Lambda_{t}-$ $\left.\sum_{k=2}^{N} p^{(k)} q^{(k)}\right)$. More explicitly, the isomonodromic evolution (2.5) of the local coordinates $p^{(k)}, q^{(k)}$ is described by

$$
\begin{aligned}
\frac{\mathrm{d} q^{(k)}}{\mathrm{d} t}= & \left(f+\frac{\Lambda_{t}-\theta_{0}^{(k)}}{t}\right) q^{(k)}+\frac{\sum_{m=1}^{N} \bar{p}^{(m)} q^{(m)}}{t-1} \\
& -\frac{\sum_{m<k} \bar{p}^{(m)} q^{(m)}}{t}-\left[\frac{\sum_{m<k} p^{(m)}}{t}+\frac{\sum_{m=1}^{N} p^{(m)}}{t(t-1)}\right]\left(q^{(k)}\right)^{2}, \\
\frac{\mathrm{d} p^{(k)}}{\mathrm{d} t}= & \left(\frac{\theta_{0}^{(k)}-\Lambda_{t}}{t}-f\right) p^{(k)}-\frac{\sum_{m=1}^{N} p^{(m)}}{t-1} \bar{p}^{(k)} \\
& +\frac{\sum_{m>k} p^{(m)}}{t} \bar{p}^{(k)}+\left[\frac{\sum_{m<k} p^{(m)}}{t}+\frac{\sum_{m=1}^{N} p^{(m)}}{t(t-1)}\right] p^{(k)} q^{(k)} .
\end{aligned}
$$

The latter system can also be rewritten in the (non-autonomous) Hamiltonian form

$$
s \frac{\mathrm{d} q^{(k)}}{\mathrm{d} s}=\frac{\partial H}{\partial p^{(k)}}, \quad s \frac{\mathrm{d} p^{(k)}}{\mathrm{d} s}=-\frac{\partial H}{\partial q^{(k)}}, \quad k=2, \ldots, N,
$$

where the Hamiltonian function is given by

$$
H=\sum_{m=1}^{N} \theta_{0}^{(m)} p^{(m)} q^{(m)}+\sum_{1 \leq m<n \leq N}\left(\bar{p}^{(m)}+p^{(m)} q^{(n)}\right) p^{(n)} q^{(m)}+\frac{1}{s-1} \sum_{m, n=1}^{N} \bar{p}^{(m)} p^{(n)} q^{(m)} .
$$

This is the Fuji-Suzuki-Tsuda system $\operatorname{FST}_{N}$. Its tau function $\tau_{\mathrm{FST}}(t)$, obtained by the appropriate specialization of the Jimbo-Miwa-Ueno tau differential (2.3), is directly related to the Hamiltonian by $s \frac{\mathrm{d}}{\mathrm{d} s} \ln \tau_{\mathrm{FST}}\left(s^{-1}\right)=H$.

In the following sections, we focus our attention on the computation of $\tau_{\mathrm{FST}}(t)$. This function is fundamental from the point of view of applications. The local coordinates on the $\mathrm{FST}_{N}$ phase space, such as $p^{(k)}, q^{(k)}$, can be expressed in terms of tau functions [29, 31] similarly to the $N=2$ case which corresponds to Painlevé VI. 


\section{Braid group dynamics of semi-degenerate monodromy}

The Riemann-Hilbert correspondence assigns to each linear system (2.1) its monodromy a point in the space $\mathcal{M}$ of conjugacy classes of triples of $G$-matrices:

$$
\mathcal{M}=\operatorname{Hom}\left(\pi_{1}(\mathbb{C} \backslash\{0,1, t\}), G\right) / G \cong\left\{\left[\left(M_{0}, M_{t}, M_{1}\right)\right], M_{0, t, 1} \in G\right\} .
$$

Local solutions of the deformation equations (2.2) continue to analytic functions of $t$ on the universal covering of $\mathbb{C} \backslash\{0,1\}$. This analytic continuation may be described [9] in terms of an action of $\mathcal{B}_{3}$, the braid group on 3 strands, on $\mathcal{M}$. Its origin may be explained as follows: the center $\mathcal{Z} \subset \mathcal{B}_{3}$ in fact acts trivially and $\mathcal{B}_{3} / \mathcal{Z} \cong \Gamma=\operatorname{PSL}(2, \mathbb{Z})$ is the mapping class group of $\mathbb{C} \backslash\{0,1, t\}$, related to the outer automorphisms of the corresponding $\pi_{1}$ by Dehn-Nielsen theorem.

To describe the braid/modular group action explicitly in a compact way, it is convenient [21] to first extend it to an action of $\bar{\Gamma}=\operatorname{PGL}(2, \mathbb{Z})$ and restrict the latter to the level 2 congruence subgroup $\bar{\Lambda}=\bar{\Gamma}(2)$. The principal gain is an extremely simple presentation of $\bar{\Lambda}$, which is freely generated by 3 involutions:

$$
\bar{\Lambda}=\left\langle x, y, z \mid x^{2}=y^{2}=z^{2}=1\right\rangle .
$$

The action of these generators on the representative triples is as follows:

$$
\begin{aligned}
& x:\left[\left(M_{0}, M_{t}, M_{1}\right)\right] \mapsto\left[\left(M_{0}^{-1}, M_{t}^{-1}, M_{t} M_{1}^{-1} M_{t}^{-1}\right)\right], \\
& y:\left[\left(M_{0}, M_{t}, M_{1}\right)\right] \mapsto\left[\left(M_{1} M_{0}^{-1} M_{1}^{-1}, M_{t}^{-1}, M_{1}^{-1}\right)\right], \\
& z:\left[\left(M_{0}, M_{t}, M_{1}\right)\right] \mapsto\left[\left(M_{0}^{-1}, M_{0} M_{t}^{-1} M_{0}^{-1}, M_{1}^{-1}\right)\right] .
\end{aligned}
$$

The words of even length in $x, y, z$ form the subgroup $\Lambda \subset \bar{\Lambda}$. It is isomorphic to free group on 2 generators (e.g., $x y$ and $y z$ ) and can be seen as a quotient $\mathcal{P}_{3} / \mathcal{Z} \cong \Lambda$ of pure braids.

Solutions of the deformation equations (2.2) with finite branching (in particular, algebraic ones) correspond to finite $\bar{\Lambda}$-orbits on $\mathcal{M}$. Of course, many examples of such orbits can be easily produced by monodromies generating finite subgroups of $\mathrm{GL}(N, \mathbb{C})$. However, their classification is an open problem except for $N=2$ [21]. In the latter case, assuming that $M_{\nu} \in \mathrm{SL}(2, \mathbb{C})$ for $\nu=0, t, 1, \infty$, the local conjugacy classes $\left[M_{\nu}\right]$ are preserved by the action of $\bar{\Lambda}$. The classification problem then reduces to the description of periodic orbits generated by 3 polynomial involutive automorphisms of an affine cubic in $\mathbb{C}^{3}$ representing the $\operatorname{SL}(2, \mathbb{C})$-character variety of the 4 punctured sphere.

We are now going to consider semi-degenerate monodromy and describe its dynamics in a more explicit form. The procedure is somewhat reminiscent of what was done on the other side of the Riemann-Hilbert correspondence in the previous subsection. Assume that $M_{0}$ and $M_{\infty}$ are diagonalizable, and $M_{1}-\mathbb{1}$ and $M_{t}-\mathbb{1}$ have rank 1 . Write

$$
M_{t}=\mathbb{1}+u^{T} \otimes v, \quad M_{1}=\mathbb{1}+\bar{u}^{T} \otimes \bar{v},
$$

and also denote

$$
\left[M_{\nu}\right]=\left[\exp \left(2 \pi \mathrm{i} \Theta_{\nu}\right)\right], \quad \nu=0,1, t, \infty, \quad \Theta_{\nu}= \begin{cases}\operatorname{diag}\left(\theta_{\nu}^{(1)}, \ldots, \theta_{\nu}^{(N)}\right), & \nu=0, \infty \\ \operatorname{diag}\left(\Lambda_{\nu}, 0, \ldots, 0\right), & \nu=t, 1 .\end{cases}
$$

In this notation, we have $v \cdot u^{T}=\mathrm{e}^{2 \pi \mathrm{i} \Lambda_{t}}-1$ and $\bar{v} \cdot \bar{u}^{T}=\mathrm{e}^{2 \pi \mathrm{i} \Lambda_{1}}-1$, which implies that $M_{t}^{-1}=\mathbb{1}-\mathrm{e}^{-2 \pi \mathrm{i} \Lambda_{t}} u^{T} \otimes v$ and $M_{1}^{-1}=\mathbb{1}-\mathrm{e}^{-2 \pi \mathrm{i} \Lambda_{1}} \bar{u}^{T} \otimes \bar{v}$. Observe that the only effect of the $\bar{\Lambda}$-action on the local monodromy exponents is the sign flip of all $\Theta_{\nu}$ for the words of odd length in $x, y, z$. 
Let us make a further assumption that $M_{0} M_{t}=\left(M_{1} M_{\infty}\right)^{-1}$ has distinct eigenvalues, different from those of $M_{0}$ and $M_{\infty}^{-1}$. Pick a representative in the conjugacy class of monodromy such that

$$
\begin{aligned}
& M_{0} M_{t}=\exp (2 \pi \mathrm{i} \mathfrak{S}), \quad \mathfrak{S}=\operatorname{diag}\left\{\sigma^{(1)}, \ldots, \sigma^{(N)}\right\}, \\
& \operatorname{Tr} \mathfrak{S}=\operatorname{Tr} \Theta_{0}+\Lambda_{t}=-\operatorname{Tr} \Theta_{\infty}-\Lambda_{1} .
\end{aligned}
$$

Lemma 3.1. For $k=1, \ldots, N$, we have

$$
\begin{aligned}
& u^{(k)} v^{(k)}=\mathrm{e}^{2 \pi \mathrm{i}\left(\Lambda_{t}-\sigma^{(k)}\right)} \frac{\prod_{l=1}^{N}\left(\mathrm{e}^{2 \pi \mathrm{i} \sigma^{(k)}}-\mathrm{e}^{2 \pi \mathrm{i} \theta_{0}^{(l)}}\right)}{\prod_{l \neq k}\left(\mathrm{e}^{2 \pi \mathrm{i} \sigma^{(k)}}-\mathrm{e}^{2 \pi \mathrm{i} \sigma^{(l)}}\right)}, \\
& \bar{u}^{(k)} \bar{v}^{(k)}=-\mathrm{e}^{-2 \pi \mathrm{i} \sigma^{(k)}} \frac{\prod_{l=1}^{N}\left(\mathrm{e}^{2 \pi \mathrm{i} \sigma^{(k)}}-\mathrm{e}^{-2 \pi \mathrm{i} \theta_{\infty}^{(l)}}\right)}{\prod_{l \neq k}\left(\mathrm{e}^{2 \pi \mathrm{i} \sigma^{(k)}}-\mathrm{e}^{2 \pi \mathrm{i} \sigma^{(l)}}\right)} .
\end{aligned}
$$

Proof. Note that

$$
\begin{aligned}
\operatorname{det}\left(\lambda-M_{0}\right) & =\operatorname{det}\left(\lambda-M_{0} M_{t}+\mathrm{e}^{-2 \pi \mathrm{i} \Lambda_{t}} M_{0} M_{t} u^{T} \otimes v\right) \\
& =\operatorname{det}\left(\lambda-M_{0} M_{t}\right)\left[1+\mathrm{e}^{-2 \pi \mathrm{i} \Lambda_{t}} v \frac{M_{0} M_{t}}{\lambda-M_{0} M_{t}} u^{T}\right] .
\end{aligned}
$$

Taking the limit as $\lambda \rightarrow \mathrm{e}^{2 \pi \mathrm{i} \sigma^{(k)}}$, we obtain the first of relations (3.4). The second is obtained by similar considerations from $\operatorname{det}\left(\lambda-M_{\infty}^{-1}\right)=\operatorname{det}\left(\lambda-M_{0} M_{t} M_{1}\right)$.

The remaining freedom of diagonal conjugation allows for rescalings

$$
\left(u^{(k)}, v^{(k)}, \bar{u}^{(k)}, \bar{v}^{(k)}\right) \mapsto\left(\alpha_{k} u^{(k)}, \alpha_{k}^{-1} v^{(k)}, \alpha_{k} \bar{u}^{(k)}, \alpha_{k}^{-1} \bar{v}^{(k)}\right),
$$

with arbitrary $\alpha_{k} \in \mathbb{C}^{*}$. It can be used, for example, to fix all $\bar{u}^{(k)}$ to be 1 and express all $\bar{v}^{(k)}$ from (3.4b). The only ambiguity which remains afterwards is the overall rescaling $(u, v) \mapsto\left(\alpha u, \alpha^{-1} v\right)$ with $\alpha \in \mathbb{C}^{*}$. This suggests to introduce the notation

$$
\sigma_{k, l}:=\sigma^{(k)}-\sigma^{(l)}, \quad s_{k, l}:=\frac{u^{(k)} \bar{u}^{(l)}}{\bar{u}^{(k)} u^{(l)}}, \quad k, l=1, \ldots, N .
$$

The local coordinates on the moduli space of semi-degenerate monodromy can be chosen as any $N-1$ independent $\sigma_{k, l}$ and $N-1$ independent $s_{k, l}$; one admissible option is to take $l=k+1$ with $k=1, \ldots, N-1$. These coordinates unambiguously fix the conjugacy class of $\left(M_{t}, M_{1}, M_{0} M_{t}\right)$.

Lemma 3.2. The involutions $x, z$ from (3.1) act as

$$
x:\left(\mathfrak{S}, s_{k l}\right) \mapsto\left(-\mathfrak{S}, s_{k l}\right), \quad z:\left(\mathfrak{S}, s_{k l}\right) \mapsto\left(-\mathfrak{S}, \mathrm{e}^{2 \pi \mathrm{i} \sigma_{k l}} s_{k l}\right) .
$$

Proof. We have

$$
x\left(\left[\left(M_{0}, M_{t}, M_{1}\right)\right]\right)=\left[\left(M_{t}^{-1} M_{0}^{-1} M_{t}, M_{t}^{-1}, M_{1}^{-1}\right)\right] .
$$

Since the product $M_{t}^{-1} M_{0}^{-1} M_{t} M_{t}^{-1}=\mathrm{e}^{-2 \pi \mathrm{i} \mathfrak{S}}$ is a diagonal matrix, it follows that $x(\mathfrak{S})=-\mathfrak{S}$. On the other hand, the transformation of the vectors $u, v, \bar{u}, \bar{v}$ can then be written as

$$
x:(u, v, \bar{u}, \bar{v}) \mapsto\left(u,-\mathrm{e}^{-2 \pi \mathrm{i} \Lambda_{t}} v, \bar{u},-\mathrm{e}^{-2 \pi \mathrm{i} \Lambda_{1}} \bar{v}\right) .
$$

Applying the same reasoning to $z\left(\left[\left(M_{0}, M_{t}, M_{1}\right)\right]\right)=\left[\left(M_{0}^{-1}, M_{0} M_{t}^{-1} M_{0}^{-1}, M_{1}^{-1}\right)\right]$, we see that

$$
z:(u, v, \bar{u}, \bar{v}) \mapsto\left(u \mathrm{e}^{2 \pi \mathrm{i} \mathfrak{S}},-\mathrm{e}^{-2 \pi \mathrm{i} \Lambda_{t}} v \mathrm{e}^{-2 \pi \mathrm{i} \mathfrak{S}}, \bar{u},-\mathrm{e}^{-2 \pi \mathrm{i} \Lambda_{1}} \bar{v}\right),
$$

which yields the second equation in (3.6). 
Remark 3.3. If the $\bar{\Lambda}$-orbit is finite, there exists an $n \in \mathbb{N}$ such that the action of $(x z)^{n}$ leaves invariant the conjugacy class of monodromy. Under the above genericity assumptions, (3.6) then implies that $\sigma_{k l} \in \mathbb{Q}$. This provides a systematic way to look for examples of finite $\bar{\Lambda}$-orbits not bounded to those coming from the finite monodromy groups. Specifically, one can set $\left[M_{0} M_{t}\right]=\left[\mathrm{e}^{2 \pi \mathrm{i} \mathfrak{S}}\right],\left[M_{0} M_{1}\right]=\left[\mathrm{e}^{2 \pi \mathrm{i} \widetilde{\mathfrak{S}}}\right]$ and perform an exhaustive computer search of orbits whose all points are characterized by $\sigma_{k l}, \bar{\sigma}_{k l} \in \mathbb{Q}$ with sufficiently small denominators. In the $N=2$ case, this procedure very quickly gives the list of all exceptional finite orbits; the proof of its completeness is quite tedious, though.

It will become clear in the next subsections that the rather simple form of the $x$ - and $z$ transformation reflects the fact that $x z$ corresponds to analytic continuation of solution of the $\mathrm{FST}_{N}$ system around the branch point $t=0$, and the coordinates $\left(\left\{\sigma_{k l}\right\},\left\{s_{k l}\right\}\right)$ on $\mathcal{M}$ are welladapted for the description of the corresponding local behavior. The form of $y$-transformation is more involved. In order to describe it more explicitly, let us first formulate an auxiliary lemma.

Lemma 3.4. Let $a=\left\{a^{(k)}\right\}_{k=1, \ldots, N}$ and $b=\left\{b^{(k)}\right\}_{k=1, \ldots, N}$ such that $a \cap b=\varnothing$ and $a^{(i)} \neq a^{(j)}$, $b^{(i)} \neq b^{(j)}$ for $i \neq j$,

$$
M_{a}=\operatorname{diag}\left\{a^{(1)}, \ldots, a^{(N)}\right\}, \quad M_{b}=\operatorname{diag}\left\{b^{(1)}, \ldots, b^{(N)}\right\},
$$

such that and $a^{(i)} \neq a^{(j)}, b^{(i)} \neq b^{(j)}$ for $i \neq j$. Define $W_{M_{a} \rightarrow M_{b}} \in \operatorname{Mat}_{N \times N}(\mathbb{C})$ by

$$
\left(W_{M_{a} \rightarrow M_{b}}\right)_{i j}=\prod_{k \neq i} \frac{a^{(j)}-b^{(k)}}{b^{(i)}-b^{(k)}}, \quad i, j=1, \ldots, N .
$$

We have

$$
\begin{gathered}
M_{b}^{-1} W_{M_{a} \rightarrow M_{b}} M_{a}\left(W_{M_{a} \rightarrow M_{b}}\right)^{-1}=\mathbb{1}+u_{M_{a}, M_{b}}^{T} \otimes v_{M_{a}, M_{b}}, \\
u_{M_{a}, M_{b}}^{(i)}=\left[b^{(i)} \prod_{k \neq i}\left(b^{(i)}-b^{(k)}\right)\right]^{-1}, \quad v_{M_{a}, M_{b}}^{(i)}=-\prod_{k}\left(b^{(i)}-a^{(k)}\right) . \\
\text { Moreover, }\left(W_{M_{a} \rightarrow M_{b}}\right)^{-1}=W_{M_{b} \rightarrow M_{a}} .
\end{gathered}
$$

Proof. The statements can be verified directly using Lagrange interpolation.

Remark 3.5. We have seen previously that, if two square matrices $M, M^{\prime}$ have disjoint simple spectra and $M^{-1} M^{\prime}-\mathbb{1}$ is of rank 1 , then the conjugacy class $\left[\left(M, M^{\prime}\right)\right]$ is uniquely determined by the eigenvalues of $M$ and $M^{\prime}$. Lemma 3.4 determines, up to diagonal factors, the explicit form of the matrix $W$ relating the eigenbases of $M$ and $M^{\prime}$. In particular,the relations (3.4a) and (3.4b) in Lemma 3.1 can be obtained as respective corollaries of $(3.8)$ with $\left(M_{a}, M_{b}\right)=\left(\mathrm{e}^{2 \pi \mathrm{i} \Theta_{0}}, \mathrm{e}^{2 \pi \mathrm{iS}}\right)$ and $\left(M_{a}, M_{b}\right)=\left(\mathrm{e}^{-2 \pi \mathrm{i} \Theta_{\infty}}, \mathrm{e}^{2 \pi \mathrm{i} \mathfrak{S}}\right)$.

More importantly, in the previous basis where $M_{0} M_{t}$ is diagonal, the matrix $M_{0}^{-1}$ can be represented as

$$
M_{0}^{-1}=D_{\mathrm{e}^{2 \pi \mathrm{i} \Theta_{0}} \rightarrow \mathrm{e}^{2 \pi \mathrm{i} \mathfrak{S}}} W_{\mathrm{e}^{2 \pi \mathrm{i} \Theta_{0}} \rightarrow \mathrm{e}^{2 \pi \mathrm{i} \mathfrak{S}}} \mathrm{e}^{-2 \pi \mathrm{i} \Theta_{0}} W_{\mathrm{e}^{2 \pi \mathrm{i} \Theta_{0}} \rightarrow \mathrm{e}^{2 \pi \mathrm{i} \mathscr{S}}}^{-1} D_{\mathrm{e}^{2 \pi \mathrm{i} \Theta_{0}} \rightarrow \mathrm{e}^{2 \pi \mathrm{i} \mathfrak{S}}}^{-1},
$$

where $W_{\mathrm{e}^{2 \pi \mathrm{i} \Theta_{0}} \rightarrow \mathrm{e}^{2 \pi \mathrm{i} \mathscr{S}}}$ is defined by $(3.7)$ and $D_{\mathrm{e}^{2 \pi \mathrm{i} \Theta_{0}} \rightarrow \mathrm{e}^{2 \pi \mathrm{i} \mathfrak{S}}}$ is a diagonal matrix whose non-zero elements can be found from the relation $M_{t}=\mathbb{1}+u^{T} \otimes v$ up to irrelevant overall scaling. One may fix them, e.g., by choosing

$$
D_{\mathrm{e}^{2 \pi \mathrm{i} \Theta_{0}} \rightarrow \mathrm{e}^{2 \pi \mathrm{i} \mathcal{S}}} u_{\mathrm{e}^{2 \pi \mathrm{i} \Theta_{0}}, \mathrm{e}^{2 \pi \mathrm{i} \mathfrak{S}}}^{T}=u^{T} .
$$

Let us now consider the matrix

$$
\mathfrak{P}:=y\left(\mathrm{e}^{2 \pi \mathrm{i} \mathfrak{S}}\right), \quad \mathfrak{P}=\operatorname{diag}\left\{\rho^{(1)}, \ldots, \rho^{(N)}\right\},
$$

defined up to permutation of its diagonal entries. 
Lemma 3.6. Suppose that $\operatorname{Spec}\left(\mathrm{e}^{-2 \pi \mathrm{i} \Theta_{0}}\right) \cap \operatorname{Spec}\left(M_{1} M_{0}^{-1} M_{1}^{-1} M_{t}^{-1}\right)=\varnothing$. The coefficients of the characteristic polynomial $\operatorname{det}(\rho-\mathfrak{P})$ can be explicitly written as rational functions in $\left\{\mathrm{e}^{2 \pi \mathrm{i} \sigma_{k l}}\right\},\left\{s_{k l}\right\}$.

Proof. The spectrum of $\mathfrak{P}$ is determined by the equation $\operatorname{det}\left(\rho-M_{1} M_{0}^{-1} M_{1}^{-1} M_{t}^{-1}\right)=0$. Under genericity assumptions of the lemma, this can be rewritten as $\operatorname{det}(\mathbb{1}+L)=0$, where

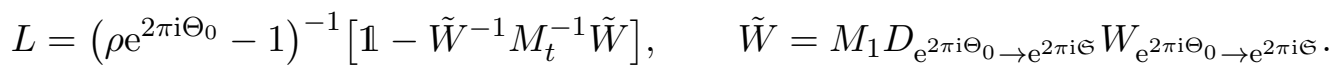

The matrix $L$ has rank 1 , which means that $\operatorname{det}(\mathbb{1}+L)=1+\operatorname{Tr} L$. The equation on the spectrum thus becomes

$$
v \tilde{W}\left(\rho \mathrm{e}^{2 \pi \mathrm{i} \Theta_{0}}-1\right)^{-1} \tilde{W}^{-1} u^{T}=-\mathrm{e}^{2 \pi \mathrm{i} \Lambda_{t}} .
$$

The left hand side of this relation contains

$$
v M_{1} D_{\mathrm{e}^{2 \pi \mathrm{i} \Theta_{0}} \rightarrow \mathrm{e}^{2 \pi \mathrm{i} \mathfrak{S}}}=v D_{\mathrm{e}^{2 \pi \mathrm{i} \Theta_{0}} \rightarrow \mathrm{e}^{2 \pi \mathrm{i} \mathfrak{S}}}+\bar{v} D_{\mathrm{e}^{2 \pi \mathrm{i} \Theta_{0}} \rightarrow \mathrm{e}^{2 \pi \mathrm{i} \mathfrak{S}}}\left(v \bar{u}^{T}\right) .
$$

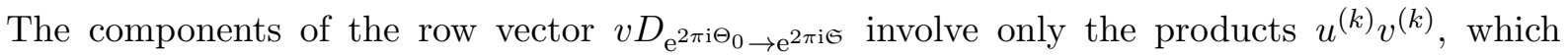
are given by (3.4a). The components of the second summand are linear combinations of $\bar{u}^{(j)} v^{(j)} u^{(k)} \bar{v}^{(k)}$, which can be expressed in terms of $\left\{s_{k l}\right\}$ by (3.5) and (3.4). Similar reasoning can be repeated for the column vector $D_{\mathrm{e}^{2 \pi \mathrm{i} \Theta_{0}}-\mathrm{e}^{2 \pi \mathrm{iS}}}^{-1} M_{1}^{-1} u^{T}$.

At last, let us explain how to compute the effect of $y$-transformation on $\left\{s_{k l}\right\}$. Denoting $\tilde{M}:=$ $M_{1} M_{0}^{-1} M_{1}^{-1} M_{t}^{-1}$, one can write $\mathrm{e}^{2 \pi \mathrm{i} \Theta_{0}} \tilde{W}^{-1} \tilde{M} \tilde{W}=\tilde{W}^{-1} M_{t}^{-1} \tilde{W}$. Applying again Lemma 3.4 with $\left(M_{\alpha}, M_{\beta}\right)=\left(\mathrm{e}^{-2 \pi \mathrm{i} \Theta_{0}}, \mathfrak{P}\right)$, we obtain

$$
\tilde{M}=\tilde{W} \tilde{D} W_{\mathfrak{P} \rightarrow \mathrm{e}^{-2 \pi \mathrm{i} \Theta_{0}}} \mathfrak{P}\left(\tilde{W} \tilde{D} W_{\mathfrak{P} \rightarrow \mathrm{e}^{-2 \pi \mathrm{i} \Theta_{0}}}\right)^{-1},
$$

where $\tilde{D}$ is the diagonal matrix determined by $\tilde{D} u_{\mathfrak{P}, \mathrm{e}^{-2 \pi \mathrm{i} \Theta_{0}}}^{T}=\tilde{W}^{-1} u^{T}$. Now from (3.1) and (3.9) it follows that we can set

$$
y\left(u^{T}\right)=W_{\mathrm{e}^{-2 \pi \mathrm{i} \Theta_{0} \rightarrow \mathfrak{P}}} u^{T}, \quad y\left(\bar{u}^{T}\right)=\left(\tilde{W} \tilde{D} W_{\mathfrak{P} \rightarrow \mathrm{e}^{-2 \pi \mathrm{i} \Theta_{0}}}\right)^{-1} \bar{u}^{T},
$$

which is sufficient to compute $\left\{s_{k l}\right\}$ from (3.5). They can therefore be expressed as rational functions of $\left\{\mathrm{e}^{2 \pi \mathrm{i} \sigma_{k l}}\right\},\left\{s_{k l}\right\}$ and $\left\{\rho^{(k)} / \rho^{(l)}\right\}$. Recall, however, that $\mathfrak{P}, \mathfrak{S}$ and $\left\{s_{k l}\right\}$ are not independent.

The above results can be used for experimental search for the finite $\bar{\Lambda}$-orbits on $\mathcal{M}$ with the help of computer algebra. However, a somewhat complicated form of the $y$-transformation is a signal that the local coordinates such as $\left\{\sigma_{k l}\right\},\left\{s_{k l}\right\}$ are not adapted to the problem of complete classification of such orbits. In addition, various special cases ruled out by our genericity assumptions should be treated separately. The most efficient approach to classification would be to find a "linearization" of the mappings (3.1), i.e., to interpret them as triples of reflections in some auxiliary (possibly infinite-dimensional?) linear space. A similar idea was successfully implemented in [9] for PVI with special local monodromy.

\section{Fredholm determinant representation of $\mathrm{FST}_{N}$ tau function}

Let us now turn to the evaluation of the $\mathrm{FST}_{N}$ tau function in terms of monodromy data. The representations of $\tau_{\mathrm{FST}}(t)$ we are dealing with in the next sections are of two types: Fredholm determinants and series over $N$-tuples of partitions. In the algebraic case, they can be expected to facilitate the reconstruction of the explicit algebraic solution curves. 
Our starting point is a topological decomposition of the isomonodromic tau function, valid for any (not necessarily semi-degenerate) 4-point Fuchsian system, see [5, equation (2.35b)]:

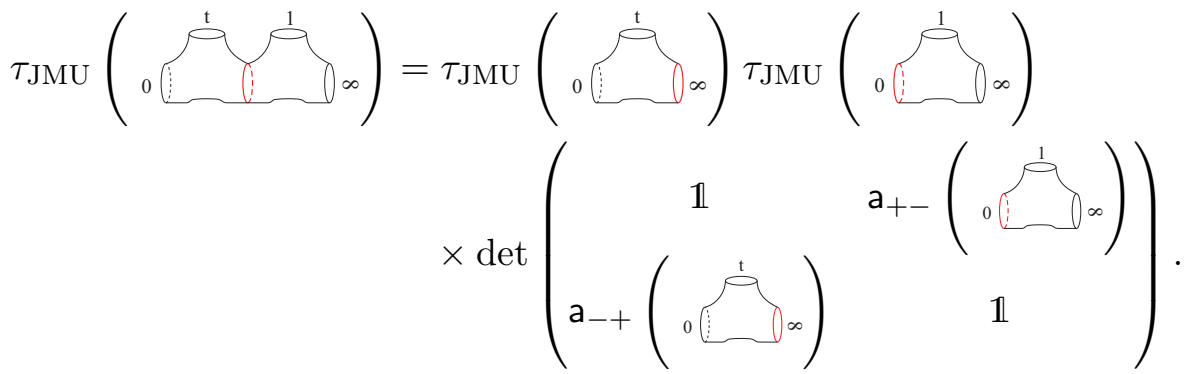

The quantities which appear on the right are associated to two auxiliary 3-point Fuchsian systems with regular singularities at $0, t, \infty$ and $0,1, \infty$, whose solutions will be denoted by $\Phi_{-}(z)$ and $\Phi_{+}(z)$. The monodromy of $\Phi_{ \pm}$is determined by matching the monodromy of the original 4-point system. If we assume for simplicity that $|t|<1$, then the matrix function $\Phi_{+}^{-1} \Phi$ (resp. $\Phi_{-}^{-1} \Phi$ ) is holomorphic and invertible inside the disk $|z|>t$ (resp. $|z|<1$ ) on $\mathbb{P}^{1}$. In particular, the monodromy of $\Phi_{-}$around $\infty$ and of $\Phi_{+}$around 0 is nothing but the composite monodromy $M_{0} M_{t}=\mathrm{e}^{2 \pi \mathrm{i} \mathfrak{S}}$ of $\Phi$.

The operators $\mathrm{a}_{ \pm \mp}$ are constructed in terms of $\Phi_{ \pm}(z)$. Consider the circle $\mathcal{C}=\{z \in \mathbb{C}$ : $|z|=R, R \in(|t|, 1)\}$ and let $H=L^{2}\left(\mathcal{C}, \mathbb{C}^{N}\right)$ be the space of vector-valued functions on $\mathcal{C}$ seen as Laurent series. Decompose this space as $H=H_{+} \oplus H_{-}$, where the subscripts \pm correspond to functions with only positive/negative modes. The operators $\mathrm{a}_{ \pm \mp}: H \rightarrow H_{ \pm}$are given by

$$
\left(\mathrm{a}_{ \pm \mp} f\right)(z)=\frac{1}{2 \pi \mathrm{i}} \oint_{\mathcal{C}} \mathrm{a}_{ \pm \mp}\left(z, z^{\prime}\right) f\left(z^{\prime}\right) \mathrm{d} z^{\prime}, \quad \mathrm{a}_{ \pm \mp}\left(z, z^{\prime}\right)= \pm \frac{\mathbb{1}-\Psi_{ \pm}(z) \Psi_{ \pm}^{-1}\left(z^{\prime}\right)}{z-z^{\prime}}
$$

where $\Psi_{ \pm}(z)=(-z)^{-\mathfrak{S}} \Phi_{ \pm}(z)$. Notice that $H_{ \pm} \subseteq$ ker $\mathrm{a}_{ \pm \mp}$, so that $\mathrm{a}_{ \pm \mp}$ can be considered as acting on $H_{\mp}$ and the determinant in (4.1) is computed on $H$. The auxiliary tau functions in (4.1) can be easily found to be $t^{\frac{1}{2} \operatorname{Tr}\left(\mathfrak{S}^{2}-\Theta_{0}^{2}-\Theta_{t}^{2}\right)}$ and 1 (here we ignore constant factors that can be chosen arbitrarily).

Consequently, whenever the inverse monodromy problem for the auxiliary 3-point Fuchsian systems can be solved, the 4-point tau function admits an explicit Fredholm determinant representation. The crucial point for us is that, when 1 of 3 singular points is semi-degenerate (which in the $\mathrm{FST}_{N}$ case is true for both $\Phi_{+}$and $\Phi_{-}$), the inverse monodromy problem does have an explicit solution in terms of generalized hypergeometric functions ${ }_{N} F_{N-1}$. Here is its construction.

Consider a semi-degenerate Fuchsian system with 3 singular points $z_{0}=0, z_{1}=1, z_{2}=\infty$. With a slight abuse of notation, we write the corresponding connection as $\partial_{z}-A(z)$ and denote by $A_{0}, A_{1}, A_{\infty}=-A_{0}-A_{1}$ its residues at the poles. Assume that $A_{0}, A_{1}, A_{\infty}$ can be represented as

$$
A_{\nu}=G_{\nu}^{-1} \Theta_{\nu} G_{\nu}, \quad \nu=0,1, \infty
$$

with $G_{0}, G_{1} \in \operatorname{GL}(N, \mathbb{C}), G_{\infty}=\mathbb{1}$ and diagonal $\Theta_{0}, \Theta_{1}, \Theta_{\infty}$ :

$$
\Theta_{\nu}=\operatorname{diag}\left(\theta_{\nu}^{(1)}, \ldots, \theta_{\nu}^{(N)}\right), \quad \nu=0, \infty, \quad \Theta_{1}=\Lambda \cdot \operatorname{diag}(1,0, \ldots, 0) .
$$

It is also assumed that all the eigenvalues of $\Theta_{0}$ and $\Theta_{\infty}$ are distinct. Such data correspond to a rigid local system and the matrix elements of $A_{1}$ are determined by $\Theta_{0}, \Theta_{\infty}$ almost uniquely:

$$
\left(R^{-1} A_{1} R\right)_{j m}=-\prod_{k}\left(\theta_{\infty}^{(j)}+\theta_{0}^{(k)}\right) \prod_{k \neq m}\left(\theta_{\infty}^{(m)}-\theta_{\infty}^{(k)}\right)^{-1}
$$


Here $R=\operatorname{diag}\left(r^{(1)}, \ldots, r^{(N)}\right)$ with all $r^{(k)} \in \mathbb{C}^{*}$ appears due to the freedom of conjugation of $A_{0,1, \infty}$ by a matrix preserving the diagonal gauge at $\infty$. The matrix $A_{1}$ has rank 1 and can therefore be written as $A_{1}=-R u^{T} \otimes v R^{-1}$, where the components of the row vectors $u$ and $v$ are given by

$$
u^{(j)}=\prod_{k}\left(\theta_{\infty}^{(j)}+\theta_{0}^{(k)}\right), \quad v^{(m)}=\prod_{k \neq m}\left(\theta_{\infty}^{(m)}-\theta_{\infty}^{(k)}\right)^{-1} .
$$

In what follows, we will need an explicit expression for the diagonalizing transformation $G_{0}=$ $\bar{R} G_{\Theta_{\infty}, \Theta_{0}} R^{-1}$, where $\bar{R}=\operatorname{diag}\left(\bar{r}^{(1)}, \ldots, \bar{r}^{(N)}\right)$ and all $\bar{r}^{(k)} \in \mathbb{C}^{*}$ can be fixed arbitrarily, together with some related formulas, cf. Lemma 3.4:

$$
\begin{aligned}
& \left(G_{\Theta_{\infty}, \Theta_{0}}\right)_{k l}=\prod_{s \neq l} \frac{\theta_{\infty}^{(s)}+\theta_{0}^{(k)}}{\theta_{\infty}^{(s)}-\theta_{\infty}^{(l)}}, \quad\left(G_{\Theta_{\infty}, \Theta_{0}}^{-1}\right)_{k l}=\prod_{s \neq l} \frac{\theta_{0}^{(s)}+\theta_{\infty}^{(k)}}{\theta_{0}^{(s)}-\theta_{0}^{(l)}}, \\
& \left(G_{\Theta_{\infty}, \Theta_{0}} u^{T}\right)_{j}=(-1)^{N-1} \prod_{k}\left(\theta_{0}^{(j)}+\theta_{\infty}^{(k)}\right), \quad\left(v G_{\Theta_{\infty}, \Theta_{0}}^{-1}\right)_{m}=\prod_{k \neq m}\left(\theta_{0}^{(k)}-\theta_{0}^{(m)}\right)^{-1} .
\end{aligned}
$$

Lemma 4.1. Let

$$
\partial_{z} \Phi(z)=\Phi(z) A(z), \quad A(z)=\frac{A_{0}}{z}+\frac{A_{1}}{z-1},
$$

be a Fuchsian system with $A_{1}$ given by (4.3) and $A_{0}+A_{1}+\Theta_{\infty}=0$.

1. The system (4.6) has a unique solution $\Phi^{(\infty)}(z)$ with the asymptotics $\Phi^{(\infty)}(z \rightarrow \infty) \simeq$ $(-z)^{-\Theta_{\infty}}\left(\mathbb{1}+O\left(z^{-1}\right)\right)$. It is explicitly given by

$$
\Phi^{(\infty)}(z)=R(-z)^{-\Theta_{\infty}} \Psi_{\Theta_{\infty}, \Theta_{0}}(1 / z) R^{-1}
$$

with

$$
\begin{aligned}
& \left(\Psi_{\Theta_{\infty}, \Theta_{0}}(1 / z)\right)_{j m} \\
& =N_{j m}^{(\infty)} z^{\delta_{j m}-1}{ }_{N} F_{N-1}\left(\begin{array}{c|c}
\left\{1-\delta_{j m}+\theta_{0}^{(k)}+\theta_{\infty}^{(j)}\right\}_{k=\overline{1, N}} & \frac{1}{z} \\
\left\{1+\theta_{\infty}^{(j)}-\theta_{\infty}^{(k)}+\delta_{m k}-\delta_{j m}\right\}_{k=1, N ; k \neq j}
\end{array}\right),
\end{aligned}
$$

where ${ }_{N} F_{N-1}$ is the generalized hypergeometric function, $j, m=1, \ldots, N$, and

$$
N_{j m}^{(\infty)}= \begin{cases}\frac{\prod_{k}\left(\theta_{\infty}^{(j)}+\theta_{0}^{(k)}\right) \prod_{k \neq m}\left(\theta_{\infty}^{(m)}-\theta_{\infty}^{(k)}\right)^{-1}}{1+\theta_{\infty}^{(j)}-\theta_{\infty}^{(m)}}, & j \neq m, \\ 1, & j=m .\end{cases}
$$

2. Similarly, there exists a unique solution $\Phi^{(0)}(z)$ of (4.6) with the normalized asymptotics $\Phi^{(0)}(z \rightarrow 0) \simeq(-z)^{\Theta_{0}}(\mathbb{1}+O(z)) G_{0}$. It can be written as

$$
\Phi^{(0)}(z)=\bar{R}(-z)^{\Theta_{0}} \Psi_{\Theta_{0}, \Theta_{\infty}}(z) G_{\Theta_{\infty}, \Theta_{0}} R^{-1}
$$

where $\Psi_{\Theta_{0}, \Theta_{\infty}}(z)$ is obtained from the previous formulas by the exchange $\Theta_{0} \leftrightarrow \Theta_{\infty}$, $z \leftrightarrow z^{-1}$, just as the notation suggests. 
3. The solutions $\Phi^{(\infty)}(z)$ and $\Phi^{(0)}(z)$ are related by $\Phi^{(0)}(z)=\bar{R} S_{\Theta_{0}, \Theta_{\infty}} R^{-1} \Phi^{(\infty)}(z)$, where the matrix elements of $S_{\Theta_{0}, \Theta_{\infty}}$ are given by

$$
\left(S_{\Theta_{0}, \Theta_{\infty}}\right)_{l j}=\prod_{k \neq l} \frac{\Gamma\left(1+\theta_{0}^{(l)}-\theta_{0}^{(k)}\right)}{\Gamma\left(1-\theta_{\infty}^{(j)}-\theta_{0}^{(k)}\right)} \prod_{k \neq j} \frac{\Gamma\left(\theta_{\infty}^{(k)}-\theta_{\infty}^{(j)}\right)}{\Gamma\left(\theta_{\infty}^{(k)}+\theta_{0}^{(l)}\right)} .
$$

The inverse transformation is obtained from $\left(S_{\Theta_{0}, \Theta_{\infty}}\right)^{-1}=S_{\Theta_{\infty}, \Theta_{0}}$.

4. The inverse matrix $\Psi_{\Theta_{\infty}, \Theta_{0}}(z)$ can be written as

$$
\Psi_{\Theta_{\infty}, \Theta_{0}}(z)^{-1}=D_{\Theta_{\infty}, \Theta_{0}} \Psi_{-\Theta_{\infty},-\Theta_{0}}(z)^{T} D_{\Theta_{\infty}, \Theta_{0}}^{-1},
$$

with $D_{\Theta_{\infty}, \Theta_{0}}=\operatorname{diag}\left\{\frac{u^{(1)}}{v^{(1)}}, \ldots, \frac{u^{(N)}}{v^{(N)}}\right\}$.

We are now prepared to formulate the main result of this section, expressing the generic $\mathrm{FST}_{N}$ tau function in terms of semi-degenerate monodromy of the associated Fuchsian system. The monodromy will be parameterized in the same as above in (3.2)-(3.3), (3.5) and will satisfy the same genericity conditions. In particular, $M_{0}, M_{\infty}$ and $M_{0} M_{t}$ are assumed diagonalizable.

Theorem 4.2. Let the pairs $\Theta_{0},-\mathfrak{S}$ and $\mathfrak{S}, \Theta_{\infty}$ have disjoint simple non-resonant spectra. The corresponding $F S T_{N}$ tau function admits a Fredholm determinant representation,

$$
\tau_{\mathrm{FST}}(t)=\mathrm{const} \cdot t^{\frac{1}{2} \operatorname{Tr}\left(\mathfrak{S}^{2}-\Theta_{0}^{2}-\Theta_{t}^{2}\right)} \operatorname{det}\left(\begin{array}{cc}
\mathbb{1} & \mathrm{a}_{+-} \\
\mathrm{a}_{-+} & \mathbb{1}
\end{array}\right),
$$

where $\mathrm{a}_{ \pm \mp}$ are integral operators defined by (4.2), with

$$
\Psi_{+}(z)=\Psi_{\mathfrak{S}, \Theta_{\infty}}(z), \quad \Psi_{-}(z)=D^{-1} t^{-\mathfrak{S}} \Psi_{-\mathfrak{S}, \Theta_{0}}\left(\frac{t}{z}\right)
$$

and $D=\operatorname{diag}\left(\exp \tilde{\beta}^{(1)}, \ldots, \exp \tilde{\beta}^{(N)}\right)$ with $\sum_{k} \tilde{\beta}^{(k)}=0$. The conjugacy class of monodromy contains the following representative, expressed in terms of $\mathfrak{S}, D$ :

$$
\begin{aligned}
& M_{0}=D^{-1} S_{-\mathfrak{S}, \Theta_{0}} \mathrm{e}^{2 \pi \mathrm{i} \Theta_{0}} S_{\Theta_{0},-\mathfrak{S}} D, \quad M_{0} M_{t}=\left(M_{1} M_{\infty}\right)^{-1}=\mathrm{e}^{2 \pi \mathrm{i} \mathfrak{S}}, \\
& M_{\infty}=S_{\mathfrak{S}, \Theta_{\infty}} \mathrm{e}^{2 \pi \mathrm{i} \Theta_{\infty}} S_{\Theta_{\infty}, \mathfrak{S}} .
\end{aligned}
$$

Remark 4.3. The last theorem extends Theorem A of [15] to higher rank $N \geq 2$. Note, however, that in [15] all monodromies are assumed to have unit determinant, whereas here we place ourselves in the gauge where the rank of $M_{t, 1}-\mathbb{1}$ is 1 . This leads to an additional elementary factor of the form $(1-t)^{\alpha}$ (called the $\mathrm{U}(1)$-factor in the gauge theory context) which should be taken into account when comparing both results for $N=2$.

\section{Series representation}

The series representation of $\tau_{\mathrm{FST}}(t)$ is obtained by expanding the determinant (4.9) into a sum of principal minors. The basic building blocks of this construction are given by the coefficients of the Fourier expansion of the kernel

$$
\begin{aligned}
\check{a}\left(z, z^{\prime}\right) & \equiv \check{a}\left(z, z^{\prime} \mid \Theta_{0}, \Theta_{\infty}\right)=\frac{\Psi_{\Theta_{\infty}, \Theta_{0}}\left(z^{-1}\right) \Psi_{\Theta_{\infty}, \Theta_{0}}\left(z^{\prime-1}\right)^{-1}-\mathbb{1}}{z-z^{\prime}} \\
& =\sum_{p, q \in \mathbb{Z}_{+}^{\prime}} \check{a}_{-q, p} z^{-q-\frac{1}{2}} z^{\prime-p-\frac{1}{2}},
\end{aligned}
$$

where $\mathbb{Z}^{\prime}=\mathbb{Z}+\frac{1}{2}$ and $\mathbb{Z}_{ \pm}^{\prime}=\mathbb{Z}_{\gtrless 0}^{\prime}$. The Fourier modes $\check{\mathrm{a}}_{-q, p}=\check{\mathrm{a}}_{-q, p}\left(\Theta_{0}, \Theta_{\infty}\right)$ are themselves $N \times N$ matrices whose entries will be denoted by $\check{a}_{-q, k ; p, l}$, with $k, l=1, \ldots, N$. 
Lemma 5.1. We have

$$
\check{\mathrm{a}}_{-q, k ; p, l}=\frac{\varphi_{-q}^{(k)} \bar{\varphi}_{p}^{(l)}}{p+q+\theta_{\infty}^{(k)}-\theta_{\infty}^{(l)}},
$$

where $\varphi_{-q}^{(k)}=\varphi_{-q}^{(k)}\left(\Theta_{0}, \Theta_{\infty}\right)$ and $\bar{\varphi}_{p}^{(l)}=\bar{\varphi}_{p}^{(l)}\left(\Theta_{0}, \Theta_{\infty}\right)$ are given by

$$
\begin{gathered}
\varphi_{-q}^{(k)}=\frac{\prod_{m=1}^{N}\left(\theta_{\infty}^{(k)}+\theta_{0}^{(m)}\right)_{q+\frac{1}{2}}}{\left(q-\frac{1}{2}\right) ! \prod_{m \neq k}^{N}\left(1+\theta_{\infty}^{(k)}-\theta_{\infty}^{(m)}\right)_{q-\frac{1}{2}}}, \\
\bar{\varphi}_{p}^{(l)}=\frac{(-1)^{N} \prod_{m=1}^{N}\left(1-\theta_{\infty}^{(l)}-\theta_{0}^{(m)}\right)_{p-\frac{1}{2}}}{\left(p-\frac{1}{2}\right) ! \prod_{m \neq l}^{N}\left(\theta_{\infty}^{(m)}-\theta_{\infty}^{(l)}\right)_{p+\frac{1}{2}}} .
\end{gathered}
$$

Proof. From the differential equation (4.6) for $\Phi^{(\infty)}(z)$ it follows that $\Psi_{\Theta_{\infty}, \Theta_{0}}\left(z^{-1}\right)$ in its turn satisfies

$$
z \partial_{z} \Psi_{\Theta_{\infty}, \Theta_{0}}\left(z^{-1}\right)=\left[\Theta_{\infty}, \Psi_{\Theta_{\infty}, \Theta_{0}}\left(z^{-1}\right)\right]+\Psi_{\Theta_{\infty}, \Theta_{0}}\left(z^{-1}\right) \frac{R^{-1} A_{1} R}{z-1} .
$$

Applying to the decomposition (5.1) the operator $z \partial_{z}+z^{\prime} \partial_{z^{\prime}}+1$ and using (5.3), we get

$$
\sum_{p, q \in \mathbb{Z}_{+}^{\prime}}\left((p+q) \check{\mathrm{a}}_{-q, p}+\left[\Theta_{\infty}, \check{\mathrm{a}}_{-q, p}\right]\right) z^{-q-\frac{1}{2}} z^{\prime-p-\frac{1}{2}}=\varphi(z) \otimes \bar{\varphi}\left(z^{\prime}\right),
$$

where $u, v$ are defined by (4.4) and

$$
\varphi(z)=\frac{\Psi_{\Theta_{\infty}, \Theta_{0}}\left(z^{-1}\right)}{z-1} u^{T}, \quad \bar{\varphi}(z)=-v \frac{\Psi_{\Theta_{\infty}, \Theta_{0}}\left(z^{-1}\right)^{-1}}{z-1} .
$$

The factorization of the right hand side of (5.4) implies that the integral operator with the kernel $\check{a}\left(z, z^{\prime}\right)$ becomes a Cauchy type matrix in the Fourier basis. Namely, the decompositions

$$
\varphi(z)=\sum_{q \in \mathbb{Z}_{+}^{\prime}} \varphi_{-q} z^{-q-\frac{1}{2}}, \quad \bar{\varphi}(z)=\sum_{p \in \mathbb{Z}_{+}^{\prime}} \bar{\varphi}_{p} z^{-p-\frac{1}{2}},
$$

yield the structure (5.2a). For the computation of Fourier components of $\varphi(z), \bar{\varphi}(z)$ observe that, taking the diagonal elements of the relation (5.3), one obtains

$$
\left(z \partial_{z} \Psi_{\Theta_{\infty}, \Theta_{0}}\left(z^{-1}\right)\right)_{k k}=-\varphi^{(k)}(z) v^{(k)} .
$$

Substituting therein the hypergeometric expressions (4.7), we immediately get the first of relations (5.2b). The explicit form of $\bar{\varphi}_{p}$ may be extracted in a similar manner from the diagonal of a counterpart of the equation (5.3) satisfied by $\Psi_{\Theta_{\infty}, \Theta_{0}}\left(z^{-1}\right)^{-1}$. Recall that this inverse can be expressed by means of (4.8).

The integral kernels $\mathrm{a}_{ \pm \mp}\left(z, z^{\prime}\right)$ of the operators appearing in the Fredholm determinant (4.9) have the structure analogous to (5.1). Decomposing them as

$$
\mathrm{a}_{ \pm \mp}\left(z, z^{\prime}\right)=\sum_{p, q \in \mathbb{Z}_{+}^{\prime}} \mathrm{a}_{ \pm q, \mp p} z^{ \pm q-\frac{1}{2}} z^{\prime \pm p-\frac{1}{2}}
$$


it follows from Theorem 4.2 (cf. equation (4.10)) that

$$
\begin{aligned}
& \mathrm{a}_{-q, k ; p, l}=\mathrm{e}^{\tilde{\beta}^{(l)}-\tilde{\beta}^{(k)}} t^{\sigma^{(l)}-\sigma^{(k)}+p+q_{\check{a}_{-q, k ; p, l}}\left(\Theta_{0},-\mathfrak{S}\right),} \\
& \mathrm{a}_{p, l ;-q, k}=\check{\mathrm{a}}_{-p, l ; q, k}\left(\Theta_{\infty}, \mathfrak{S}\right),
\end{aligned}
$$

where ă's are explicitly given by Lemma 5.1.

The principal minor expansion of the determinant (4.9) can now be calculated following the scheme outlined in [5, Section 2.2.1]. We obtain

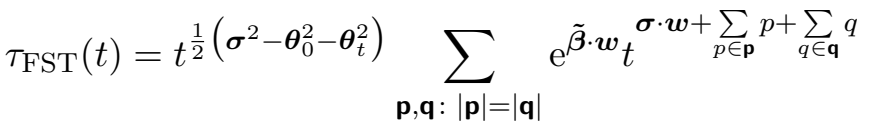

$$
\begin{aligned}
& \times(-1)^{|\mathbf{p}|} \operatorname{det} \check{\mathbf{a}}_{-\mathbf{q}, \mathbf{p}}\left(\Theta_{0},-\mathfrak{S}\right) \operatorname{det} \check{a ̆}_{-\mathbf{p}, \mathbf{q}}\left(\Theta_{\infty}, \mathfrak{S}\right) \text {. }
\end{aligned}
$$

The notation is as follows:

- $\mathbf{p}=\mathrm{p}^{(1)} \sqcup \cdots \sqcup \mathrm{p}^{(N)}, \mathbf{q}=\mathrm{q}^{(1)} \sqcup \cdots \sqcup \mathbf{q}^{(N)}$, where each of $\mathbf{p}^{(k)}, \mathbf{q}^{(k)}$ is a finite subset of $\mathbb{Z}_{+}^{\prime}$. The elements of $\mathrm{p}^{(k)}$ and $-\mathrm{q}^{(k)}$ may be considered as positions of particles and holes in a Maya diagram of color $k \in\{1, \ldots, N\}$. The summation in (5.5) is carried over $N$-tuples of Maya diagrams satisfying the condition of global balance: $\sharp$ (particles $)=\sharp($ holes $)$.

- $\boldsymbol{w}=\left(w^{(1)}, \ldots, w^{(N)}\right)$, where $w^{(k)}=\left|\mathbf{p}^{(k)}\right|-\left|\mathbf{q}^{(k)}\right|$ is the charge of the appropriate Maya diagram. Obviously, we have $\sum_{k=1}^{N} w^{(k)}=0$, i.e., $\boldsymbol{w}$ belongs to the $\mathfrak{s l}_{N}$ root lattice, to be denoted by $\mathfrak{Q}_{N-1}$. We denote $\boldsymbol{\sigma}=\left(\sigma^{(1)}, \ldots, \sigma^{(N)}\right), \tilde{\boldsymbol{\beta}}=\left(\tilde{\beta}^{(1)}, \ldots, \tilde{\beta}^{(N)}\right)$ etc., so that, for instance, $\boldsymbol{\sigma} \cdot \boldsymbol{w}=\sum_{k=1}^{N} w^{(k)} \sigma^{(k)}$ and $\operatorname{Tr} \mathfrak{S}^{2}=\boldsymbol{\sigma}^{2}$.

- $\check{a}_{-\mathbf{q}, \mathbf{p}}$ are $|\mathbf{p}| \times|\mathbf{p}|$ matrices obtained by restriction of ă written in the Fourier basis to rows and columns labeled by $-\mathbf{q}$ and $\mathbf{p}$.

- Using the correspondence between Maya diagrams and charged Young diagrams, the sum in (5.5) can be alternatively rewritten as $\sum_{\boldsymbol{w} \in \mathfrak{Q}_{N-1}} \sum_{\boldsymbol{Y} \in \mathbb{Y}^{N}}$, where $\mathbb{Y}$ denotes the set of all Young diagrams. The reader is referred to $[5,15,16]$ for the details.

Let us stress that the structure of (5.5) is not specific for semi-degenerate monodromy. The spectral constraints played a role above only in finding the explicit form of the 3-point solutions $\Psi_{ \pm}(z)$ and matrix elements $\check{a ̆}_{-q, p}$ in Lemma 5.1. An additional bonus is the Cauchy structure (5.2a) of these matrix elements which enables one to calculate the determinants in (5.5) in a factorized form. In the generic situation, one has a sum of $N-1$ Cauchy matrices instead of just 1 .

\section{Scalar ${ }_{N} \boldsymbol{F}_{N-1}$ kernel}

It was shown in [15] that, for special monodromy data, the tau function of the Painlevé VI equation $\left(\mathrm{FST}_{2}\right.$ system) can be expressed as a scalar Fredholm determinant on the interval $\mathcal{B}:=$ $[0, t] \subset \mathbb{R}$. The relevant integrable ${ }_{2} F_{1}$ kernel is related to the so-called ZW-measures [4]. Here we generalize the former result to $N>2$ by constructing a class of $\operatorname{FST}_{N}$ tau functions which can be represented by scalar Fredholm determinants with an integrable ${ }_{N} F_{N-1}$ kernel on $\mathcal{B}$.

We denote $M_{0} M_{t}=\mathrm{e}^{2 \pi \mathrm{i} S}, \mathfrak{S}=\operatorname{diag}\left\{\sigma^{(1)}, \ldots, \sigma^{(N)}\right\}$ as before, and consider a special monodromy for the inner auxiliary 3 -point problem with singular points $0, t, \infty$ :

$$
M_{t}=1+\mathrm{e}^{-\mathrm{i} \pi \mathfrak{S}} u^{T} \otimes v \mathrm{e}^{\mathrm{i} \pi \mathfrak{S}} .
$$


Here the row vectors $u$ and $v$ are defined by

$$
u^{T}=2 \pi \mathrm{i} \sum_{i \in I} s_{i} e_{i}, \quad v=\sum_{j \in J} s_{j}^{-1} e^{j},
$$

and $I, J$ are two non-empty non-intersecting subsets of all indices:

$$
I, J \subset\{1, \ldots, N\}, \quad I \cap J=\varnothing .
$$

The parameters $s_{i}$ are non-zero complex numbers and $e_{i}$ are the standard basis vectors in $\mathbb{C}^{N}$. Due to (6.2) and (6.3), one has $v \cdot u^{T}=0$. From the explicit expression for $M_{t}$ it follows that the spectra of $M_{0}$ and $M_{0} M_{t}$ coincide.

The monodromy matrix $M_{t}$ is non-diagonalizable. Therefore, Theorem 4.2 together with the previous parameterization of 3-point Fuchsian systems are not directly applicable. Nevertheless it is not difficult to guess the form of a 3-point Fuchsian system having the prescribed monodromy (6.1):

$$
\frac{\mathrm{d} \Phi_{-}(z)}{\mathrm{d} z}=\Phi_{-}(z)\left[\frac{\mathfrak{S}}{z}+\frac{u^{T} \otimes v}{2 \pi \mathrm{i}(z-t)}\right] .
$$

Rewriting this equation in terms of a new function $\chi(z)=\Phi_{-}(t z)(-z)^{-\mathfrak{S}}$, one obtains

$$
\frac{\mathrm{d} \chi(z)}{\mathrm{d} z}=\chi(z) \frac{(-z)^{\mathfrak{S}} u^{T} \otimes v(-z)^{-\mathfrak{S}}}{2 \pi \mathrm{i}(z-1)} .
$$

Its solution (normalized at $z=\infty$ ) can be written as

$$
\chi(z)=\mathbb{1}+\sum_{i \in I, j \in J} \frac{(-z)^{\sigma^{(i)}-\sigma^{(j)}} l_{\sigma^{(j)}-\sigma^{(i)}}(1 / z)}{\sigma^{(i)}-\sigma^{(j)}} s_{i} s_{j}^{-1} e_{i} \otimes e^{j},
$$

where

$$
l_{\sigma}(z)={ }_{2} F_{1}\left(\begin{array}{c}
1, \sigma \\
1+\sigma
\end{array} \mid z\right) .
$$

This function is naturally defined on $\mathbb{C} \backslash \mathbb{R}_{\geq 1}$. We are going to use a jump property of the rescaled function $l_{\sigma}\left(\frac{t}{z}\right)$ which can be derived from $(6.5)$ :

$$
l_{\sigma}\left(\frac{t}{x+\mathrm{i} 0}\right)-l_{\sigma}\left(\frac{t}{x-\mathrm{i} 0}\right)=-2 \pi \mathrm{i} \sigma\left(\frac{x}{t}\right)^{\sigma}, \quad 0<x<t .
$$

The structure of analytic continuation of $\chi(z)$ follows from the analytic properties of $l_{\sigma}(z)$. For example, the continuation of $\chi(z)$ along the curve $\gamma_{1}$ encircling $z=1$ in the anti-clockwise direction gives

$$
\chi\left(\gamma_{1} . z\right)=\chi(z)+\mathrm{e}^{-\mathrm{i} \pi \mathfrak{S}} u^{T} \otimes v \mathrm{e}^{\mathrm{i} \pi \mathfrak{S}}=\left(\mathbb{1}+\mathrm{e}^{-\mathrm{i} \pi \mathfrak{S}} u^{T} \otimes v \mathrm{e}^{\mathrm{i} \pi \mathfrak{S}}\right) \chi(z),
$$

where we used the jump relation (6.6) for $t=1$ and the nilpotency properties. Therefore, the monodromy of $\Phi_{-}(z)=\chi\left(\frac{z}{t}\right)\left(-\frac{z}{t}\right)^{\mathfrak{S}}$ is given by

$$
\Phi_{-}\left(\gamma_{t} \cdot z\right)=\left(\mathbb{1}+\mathrm{e}^{-\mathrm{i} \pi \mathfrak{S}} u^{T} \otimes v \mathrm{e}^{\mathrm{i} \pi \mathfrak{S}}\right) \Phi_{-}(z), \quad \Phi_{-}\left(\gamma_{\infty}^{-1} \cdot z\right)=\mathrm{e}^{2 \pi \mathrm{i} \mathfrak{S}} \Phi_{-}(z) .
$$

Thus $\Phi_{-}(z)$ solves the Fuchsian system (6.4) and has prescribed monodromy. Below we also need the explicit expression for $\Psi_{-}(z)=(-z)^{-\mathfrak{S}} \Phi_{-}(z)$ :

$$
\Psi_{-}(z)=t^{-\mathfrak{S}}\left(\mathbb{1}+\sum_{i \in I, j \in J} \frac{l_{\sigma^{(j)}-\sigma^{(i)}\left(\frac{t}{z}\right)}}{\sigma^{(i)}-\sigma^{(j)}} s_{i} s_{j}^{-1} e_{i} \otimes e^{j}\right) .
$$


Let us investigate the integral kernels of the operators $a_{-+}$and $a_{+-}$appearing in the Fredholm determinant representation of the tau function, $\tau(t)=\operatorname{det}\left(\mathbb{1}-\mathrm{a}_{+-} \mathrm{a}_{-+}\right)$, and given by (4.2). In the initial setting, $a_{ \pm \mp}$ acted on functions on a circle $\mathcal{C}$ centered at $z=0$ and having radius $R \in(t, 1)$. Let us now shrink the contour $\mathcal{C}$ to the branch cut $\mathcal{B}$ of $\Psi_{-}(z)$, and in this way transform $L^{2}\left(S^{1}, \mathbb{C}^{N}\right) \leadsto \mathcal{W}=L^{2}\left(\mathcal{B}, \mathbb{C}^{N}\right)_{\text {up }} \oplus L^{2}\left(\mathcal{B}, \mathbb{C}^{N}\right)_{\text {down }}=\mathcal{W}_{s} \oplus \mathcal{W}_{a}$, where $s$ and $a$ stand for symmetric and antisymmetric functions with respect to exchange of their boundary values on the two sides of $\mathcal{B}$ :

$$
\begin{aligned}
& \mathcal{W}_{s}=\{f \in \mathcal{W}: f(x+\mathrm{i} 0)=+f(x-\mathrm{i} 0), x \in \mathcal{B}\} \\
& \mathcal{W}_{a}=\{f \in \mathcal{W}: f(x+\mathrm{i} 0)=-f(x-\mathrm{i} 0), x \in \mathcal{B}\}
\end{aligned}
$$

Since $\Psi_{+}(z)$ has no jump on $\mathcal{B}$ symmetric functions contain the image and belong to the kernel of $a_{+-}$:

$$
\left(\mathrm{a}_{+-}\right)_{s s}=\left(\mathrm{a}_{+-}\right)_{a s}=\left(\mathrm{a}_{+-}\right)_{a a}=0 .
$$

Consequently, the only part of $\mathrm{a}_{-+}$important for the determinant computation is $\left(\mathrm{a}_{-+}\right)_{a s}$.

Using the evaluation (6.7) and the jump property (6.6) of $l_{\sigma}\left(\frac{t}{z}\right)$, it is straightforward to obtain formulas for the jumps of $\Psi_{-}(z)$ and $\Psi_{-}^{-1}(z)$ on $\mathcal{B}$. For $x, y \in(0, t)$, one has

$$
\begin{aligned}
& \Psi_{-}(x+\mathrm{i} 0)-\Psi_{-}(x-\mathrm{i} 0)=x^{-\mathfrak{S}} u^{T} \otimes v x^{\mathfrak{S}} t^{-\mathfrak{S}}, \\
& \Psi_{-}^{-1}(y+\mathrm{i} 0)-\Psi_{-}^{-1}(y-\mathrm{i} 0)=-t^{\mathfrak{S}} y^{-\mathfrak{S}} u^{T} \otimes v y^{\mathfrak{S}} .
\end{aligned}
$$

Lemma 6.1. Let $\max _{i, j}\left|\Re\left(\sigma^{(i)}-\sigma^{(j)}\right)\right|<1$. For $n \in \mathbb{Z}_{\geq 0}$, define $X_{n}:=\operatorname{Tr}\left(\mathrm{a}_{+-} \mathrm{a}_{-+}\right)^{n}$. We have the equality

$$
X_{n}=\operatorname{Tr} K^{n},
$$

where $K$ is an integral operator on $L^{2}(\mathcal{B})$ with the kernel

$$
K(x, y)=-\sum_{i \in I, j \in J} s_{j}^{-1} x^{\sigma^{(j)}} \frac{\left(\Psi_{+}(x) \Psi_{+}(y)^{-1}\right)_{j i}}{x-y} y^{-\sigma^{(i)}} s_{i} .
$$

Proof. Let $g \in L^{2}\left(\mathcal{B}, \mathbb{C}^{N}\right)$. The action of $\mathrm{a}_{-+}$on $f=g \oplus g \in \mathcal{W}_{s}$ reads

$$
\begin{aligned}
\left(\mathrm{a}_{-+} f\right)(z) & =\frac{1}{2 \pi \mathrm{i}} \int_{0}^{t} \frac{\Psi_{-}(z)\left[\Psi_{-}^{-1}(y-\mathrm{i} 0)-\Psi_{-}^{-1}(y+\mathrm{i} 0)\right]}{z-y} g(y) \mathrm{d} y \\
& =\frac{1}{2 \pi \mathrm{i}} \int_{0}^{t} \frac{y^{-\mathfrak{S}} u^{T} \otimes v y^{\mathfrak{S}}}{z-y} g(y) \mathrm{d} y, \quad z \notin \mathcal{B},
\end{aligned}
$$

where at the second step we used the relation (6.8) and nilpotency. The next step is to compute the projection $\Pi_{a}$ of this expression on $\mathcal{W}_{a}$. Write the result as $\Pi_{a} \circ \mathrm{a}_{-+} f=h \oplus(-h)$, with some $h \in L^{2}\left(\mathcal{B}, \mathbb{C}^{N}\right)$. For $x \in \mathcal{B}$, one has

$$
\begin{aligned}
h(x) & =\frac{1}{2}\left[\left(\mathrm{a}_{-+} f\right)(x+\mathrm{i} 0)-\left(\mathrm{a}_{-+} f\right)(x-\mathrm{i} 0)\right] \\
& =-\frac{1}{4 \pi i} \int_{0}^{t} y^{-\mathfrak{S}} u^{T} \otimes v y^{\mathfrak{S}}\left(\frac{1}{y-x-\mathrm{i} 0}-\frac{1}{y-x+\mathrm{i} 0}\right) g(y) \mathrm{d} y \\
& =-\frac{1}{2} x^{-\mathfrak{S}} u^{T} \otimes v x^{\mathfrak{S}} g(x) .
\end{aligned}
$$


At last, write $\mathrm{a}_{+-} \circ \Pi_{a} \circ \mathrm{a}_{-+} f$ as $\tilde{g} \oplus \tilde{g} \in \mathcal{W}_{s}$. The expression (6.11) for $h(x)$ implies that

$$
\tilde{g}(x)=\frac{1}{2 \pi \mathrm{i}} \int_{0}^{t} \mathrm{a}_{+-}(x, y) y^{-\mathfrak{S}} u^{T} \otimes v y^{\mathfrak{S}} g(y) \mathrm{d} y, \quad x \in \mathcal{B} .
$$

The last formula describes the action of $\mathrm{a}_{+-} \circ \Pi_{a} \circ \mathrm{a}_{-+}$on $\mathcal{W}_{s}$. To obtain (6.9)-(6.10), it now suffices to compute the $n$th power of this operator, use the cyclic property of the trace and the explicit form (4.2) of the integral kernel $\mathrm{a}_{+-}(x, y)$.

We now summarize the developments of this section:

Theorem 6.2. Let $I, J$ be two non-intersecting subsets of $\{1, \ldots, N\}$ and let

$$
\left\{\theta_{\infty}^{(k)}\right\}_{k=\overline{1, N}}, \quad\left\{\sigma^{(k)}\right\}_{k=\overline{1, N}}, \quad\left\{s_{i}\right\}_{i \in I \cup J}
$$

be complex parameters such that

- $s_{i} \neq 0$ for $i \in I \cup J$ and $\sigma^{(i)} \neq \sigma^{(j)}$ for $i \neq j$;

- $\max _{i, j \in\{1, \ldots, N\}}\left|\Re\left(\sigma^{(i)}-\sigma^{(j)}\right)\right|<1$.

Let $K$ be the integral operator on $L^{2}([0, t])$ with the integrable kernel $(6.10)$, where

$$
\begin{aligned}
& \left(\Psi_{+}(x)\right)_{j m}=C_{j m} x^{1-\delta_{j m}{ }_{N} F_{N-1}}\left(\begin{array}{c}
\left\{1-\delta_{j m}+\theta_{\infty}^{(k)}+\sigma^{(j)}\right\}_{k=\overline{1, N}} \\
\left\{1+\sigma^{(j)}-\sigma^{(k)}+\delta_{m k}-\delta_{j m}\right\}_{k=\overline{1, N}, k \neq j}
\end{array} \mid x\right), \\
& \left(\Psi_{+}(y)^{-1}\right)_{m i}=C_{m i}^{\prime} y^{1-\delta_{i m}} F_{N-1}\left(\begin{array}{c}
\left\{1-\delta_{i m}-\theta_{\infty}^{(k)}-\sigma^{(i)}\right\}_{k=\overline{1, N}} \\
\left\{1-\sigma^{(i)}+\sigma^{(k)}+\delta_{m k}-\delta_{i m}\right\}_{k=\overline{1, N}, k \neq i}
\end{array} \mid y\right), \\
& C_{j m}=\frac{\prod_{k=1}^{N}\left(\sigma^{(j)}+\theta_{\infty}^{(k)}\right)}{\left(\sigma^{(j)}-\sigma^{(m)}+1\right) \prod_{k \neq m}\left(\sigma^{(m)}-\sigma^{(k)}\right)}=-C_{m j}^{\prime} \quad \text { for } j \neq m, \\
& C_{m m}=C_{m m}^{\prime}=1 .
\end{aligned}
$$

Then the scalar Fredholm determinant $\tau(t)=\operatorname{det}\left(\mathbb{1}-K_{[0, t]}\right)$ is a tau function of the FST system with $\Lambda_{t}=0$.

Remark 6.3. Note that since $\Psi_{+}(x) \Psi_{+}(x)^{-1}=\mathbb{1}$, the kernel $K(x, y)$ is not singular along the diagonal $x=y$. The formulas (6.12) follow from the identification $\Psi_{+}(x)=\Psi_{\mathfrak{S}, \Theta_{\infty}}(x)$, cf. equation (4.7) of Lemma 4.1; in particular, (6.12b) is consistent with the inversion formula (4.8). The semi-degenerate spectral type of the Fuchsian singularity at $z=1$ was only necessary to obtain explicit expression of the auxiliary 3-point solution $\Psi_{+}(z)$. The rest of the argument remains valid even if the singularity at $z=1$ is generic.

\section{Multivariate extension}

This section is devoted to a multivariate generalization of the $\mathrm{FST}_{N}$ system obtained by adding extra semi-degenerate singularities. The matrix $A(z)$ in $(2.1)$ is replaced by

$$
A(z)=\sum_{k=0}^{n-2} \frac{A_{k}}{z-z_{k}}, \quad z_{0}=0, \quad z_{n-2}=1
$$


where $A_{1}, \ldots, A_{n-2}$ have rank 1 . We set $z_{n-1}=\infty, A_{n-1}=-\sum_{k=0}^{n-2} A_{k}$ and assume radial ordering: $0<\left|z_{1}\right|<\cdots<\left|z_{n-3}\right|<1$. The fundamental solution $\Phi(z)$ has monodromy $M_{k} \in \mathrm{GL}(N, \mathbb{C})$ upon analytic continuation around $z_{k}(k=0, \ldots, n-1)$, see Fig. 1 for the $n=4$ case. These monodromy matrices satisfy the cyclic relation $M_{0} \cdots M_{n-1}=\mathbb{1}$. It will be convenient for us to consider the products $M_{0 \rightarrow k}:=M_{0} \cdots M_{k}$ and introduce a notation for their spectrum via $\left[M_{0 \rightarrow k}\right]=\left[\mathrm{e}^{2 \pi \mathrm{i} \mathfrak{S}_{k}}\right], k=0, \ldots, n-2$, where the eigenvalues of diagonal matrices $\mathfrak{S}_{k}$ are assumed to be pairwise distinct $\bmod \mathbb{Z}$. It may also be assumed that $\operatorname{Tr} \mathfrak{S}_{k}=\sum_{j=0}^{k} \operatorname{Tr} \Theta_{j}$. For notational purposes, it is convenient to identify $\mathfrak{S}_{0}=\Theta_{0}, \mathfrak{S}_{n-2}=-\Theta_{n-1}$.

Remark 7.1. The solution of the multivariate extension of the $\mathrm{FST}_{N}$ system corresponding to reducible monodromy (i.e., Riccati-type solution) was constructed in terms of a multivariate hypergeometric series in $[22,30]$. Our aim in this section is to study the solutions corresponding to generic semi-degenerate monodromy. One of their potential applications is the theory of Frobenius manifolds, where isomonodromic deformations of the Fuchsian systems with degenerate local monodromies naturally arise, see for example [8, Section 3] and [6, Section 8].

The following straightforward generalization of Lemma 3.1 provides a parameterization of semi-degenerate monodromy [14].

Proposition 7.2. Let $M_{k} \in \mathrm{GL}(N, \mathbb{C})$ with $k=0, \ldots, n-1$ be the monodromy matrices of the semi-degenerate Fuchsian system satisfying the above genericity condition. Given their spectra $\left[M_{k}\right]=\left[\exp 2 \pi \Theta_{k}\right]$, they can be parameterized uniquely (up to an overall conjugation) by means of diagonal matrices $\left\{\mathfrak{S}_{k}, D_{k}\right\}$ with $k=1, \ldots, n-3$, where $D_{k}=\operatorname{diag}\left\{\mathrm{e}^{\beta_{k}^{(1)}}, \ldots, \mathrm{e}^{\beta_{k}^{(N)}}\right\}, \beta_{k}^{(l)} \in \mathbb{C}$. The parametrization of $M_{k}=\left(M_{0 \rightarrow k-1}\right)^{-1} M_{0 \rightarrow k}$ follows from

$$
\begin{aligned}
& M_{0 \rightarrow k}=S_{k} \mathrm{e}^{2 \pi \mathrm{i} \mathfrak{S}_{k}} S_{k}^{-1}, \quad k=0, \ldots, n-2, \\
& S_{k}^{-1}=D_{k} W_{k+1} D_{k+1} \cdots D_{n-3} W_{n-2} D_{n-2},
\end{aligned}
$$

where

$$
\left(W_{m}\right)_{k l}=\frac{\prod_{s \neq k} \sin \pi\left(\sigma_{m-1}^{(s)}-\sigma_{m}^{(l)}\right)}{\prod_{s \neq l} \sin \pi\left(\sigma_{m}^{(l)}-\sigma_{m}^{(s)}\right)} .
$$

For $n>4$, the circle $\mathcal{C}$ of the 4-point system is replaced with $n-3$ non-intersecting simple closed curves decomposing $\mathbb{C P}^{1} \backslash\left\{z_{0}, \ldots, z_{n-1}\right\}$ into $n-2$ spheres with 3 punctures. A general method to construct Fredholm determinant representation of the isomonodromic tau function in the multi-curve setup is outlined in [5]. Among different topologically inequivalent systems of cutting curves, we are going to use the simplest one, given by a set of concentric cirles

$$
\mathcal{C}_{k}=\left\{z \in \mathbb{C}:|z|=R_{k},\left|z_{k}\right|<R_{k}<\left|z_{k+1}\right|\right\}, \quad k=1, \ldots, n-3,
$$

and corresponding to a linear pants decomposition, cf. [5, Section 3.3]. Note that the diagonal matrices $\left\{\mathfrak{S}_{k}, D_{k}\right\}$ may be thought of as associated with the circle $\mathcal{C}_{k}$. The analog of (4.1) is given by

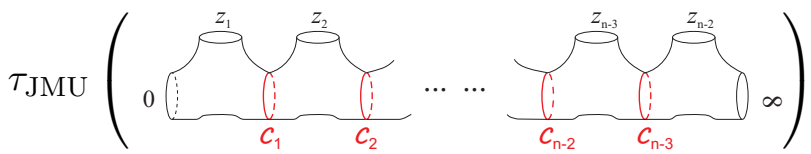




$$
=\prod_{k=1}^{n-2} \tau_{\mathrm{JMU}}(\underbrace{\sum_{c_{k}}^{z_{k}}}_{c_{k-1}} \underbrace{}_{\infty}) \operatorname{det}\left(\begin{array}{cc}
\mathbb{1} & \mathrm{A}_{+--} \\
\mathrm{A}_{-+} & \mathbb{1}
\end{array}\right),
$$

where

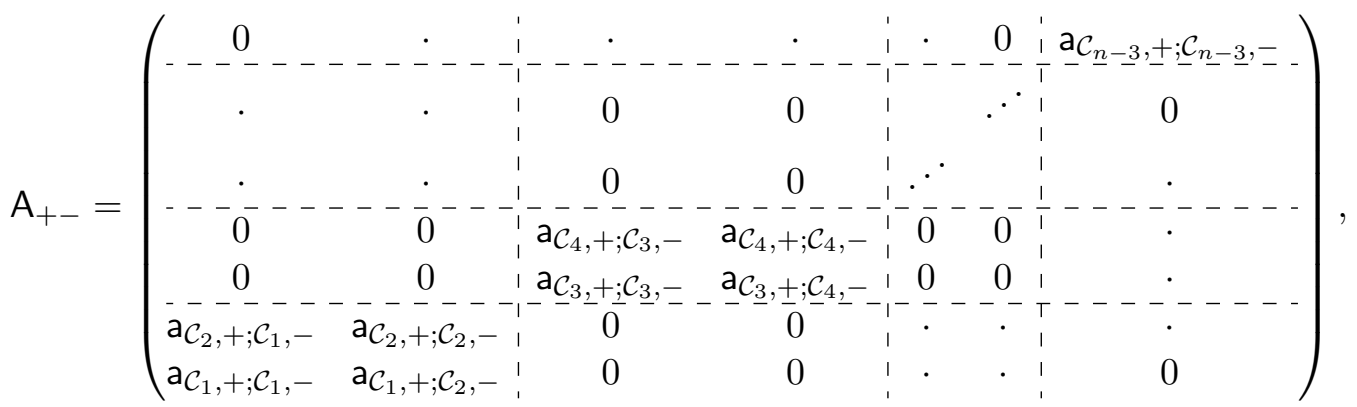

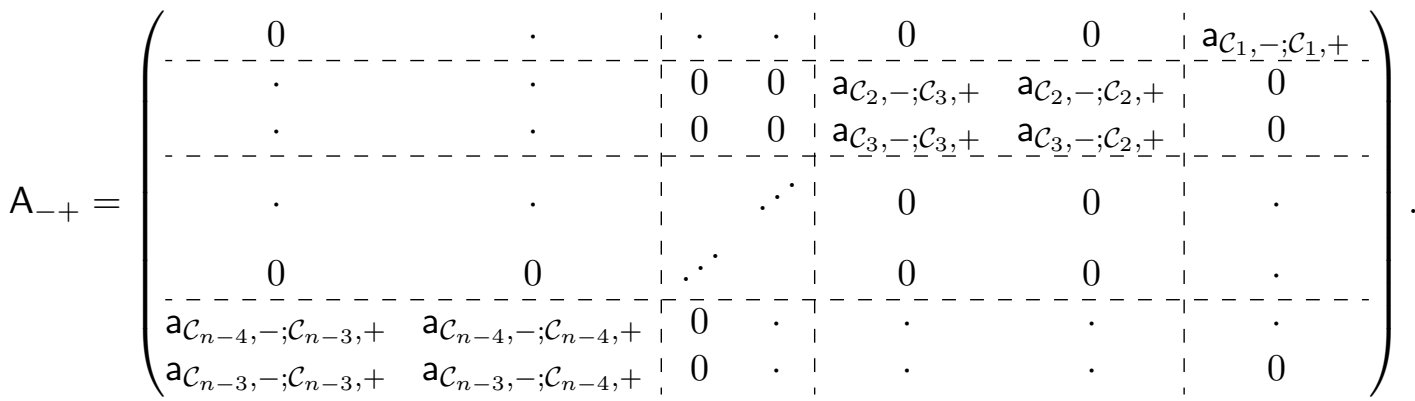

A few explanations are in order:

- The tau functions of auxiliary 3-point Fuchsian systems have elementary expressions in terms of monodromy of the initial $n$-point problem:

$$
\tau_{\mathrm{JMU}}(\underbrace{\overbrace{c_{\mathrm{k}}}^{z_{\mathrm{k}}}}_{c_{\mathrm{k}-1}} \underbrace{}_{\infty})=z_{k}^{\frac{1}{2} \operatorname{Tr}\left(\mathfrak{S}_{k}^{2}-\mathfrak{S}_{k-1}^{2}-\Theta_{k}^{2}\right)} .
$$

Recall that $\mathfrak{S}_{k}$ and $\Theta_{k}$ are diagonal matrices such that $\left[M_{k}\right]=\left[\mathrm{e}^{2 \pi \mathrm{i} \Theta_{k}}\right]$ and $\left[M_{0} \cdots M_{k}\right]=$ $\left[\mathrm{e}^{2 \pi \mathrm{i} \mathfrak{S}_{k}}\right]$, where $M_{k}$ denotes the anti-clockwise monodromy around $z_{k}$.

- The structure of the operators $A_{ \pm \mp}$ is determined by the choice of the pants decomposition and its 2-coloring. We fix them in the same way as in [5, Section 3.3]. The pants with boundary components $\mathcal{C}_{k-1}$ and $\mathcal{C}_{k}$ will be denoted by $\mathcal{T}^{[k]}$. The odd- and evennumbered pairs of pants will have color "+" and "-". The orientation of the circles is fixed accordingly: $\mathcal{C}_{2 k-1}$ and $\mathcal{C}_{2 k}$ are oriented anti-clockwise and clockwise, respectively.

- The rows and columns of $A_{ \pm \mp}$ are labeled by the curves $\mathcal{C}_{1}, \ldots, \mathcal{C}_{n-3}$. The element of $A_{ \pm \mp}$ corresponding to a pair $\mathcal{C}_{k}, \mathcal{C}_{l}$ is non-zero only if the two curves are boundary components of the same pair of pants of color "干". The formulas (7.3) correspond thus to even $n$. For odd $n$ the block in the upper-right corner of $\mathrm{A}_{+-}$would be $2 \times 2$, whereas the block in the bottom-left corner of $A_{-+}$would be $1 \times 1$.

Denote $H_{\mathcal{C}}=L^{2}\left(\mathcal{C}, \mathbb{C}^{N}\right)$ and let $H_{\mathcal{C}, \pm}$ be the space of boundary values of functions that continue analytically to the interior/exterior of $\mathcal{C}$ with respect to its orientation. The determinant in (7.2) is computed on $H=\bigoplus_{k=1}^{n-3} H_{\mathcal{C}_{k}}$. The operators a $\mathrm{C}_{, \pm ; \mathcal{C}^{\prime}, \mp}$ act from $H_{\mathcal{C}^{\prime}, \mp}$ to $H_{\mathcal{C}, \pm}$ as follows:

$$
\left(\mathrm{a}_{\mathcal{C}, \pm ; \mathcal{C}^{\prime}, \mp} g\right)(z)=\frac{1}{2 \pi \mathrm{i}} \oint_{\mathcal{C}^{\prime}} \mathrm{a}_{\mathcal{C}, \pm ; \mathcal{C}^{\prime}, \mp}\left(z, z^{\prime}\right) g\left(z^{\prime}\right) \mathrm{d} z^{\prime}, \quad z \in \mathcal{C},
$$


with the integral kernel

$$
\mathrm{a}_{\mathcal{C}, \pm ; \mathcal{C}^{\prime}, \mp}\left(z, z^{\prime}\right)= \pm \frac{\Psi_{\mathcal{C}, \pm}(z) \Psi_{\mathcal{C}^{\prime}, \pm}\left(z^{\prime}\right)^{-1}-\mathbb{1} \delta_{\mathcal{C}, \mathcal{C}^{\prime}}}{z-z^{\prime}}
$$

The functions $\Psi_{\mathcal{C}, \pm}(z), \Psi_{\mathcal{C}^{\prime}, \pm}(z)$ are determined by the fundamental solutions of the auxiliary 3 -point Fuchsian systems associated to appropriate pairs of pants, see for instance [5, equations (3.9)]. Note that each $2 \times 2$ block of $A_{ \pm \mp}$ involves only one such 3 -point solution; the blocks could thus be labeled by $\mathcal{T}^{[k]}$.

Denote by $\Phi^{[k]}(z)(k=1, \ldots, n-2)$ the solution of 3-point Fuchsian system associated with the pants $\mathcal{T}^{[k]}$ which has regular singularities at $0, z_{k}$ and $\infty$ characterized by monodromies $M_{0 \rightarrow k-1}, M_{k}$ and $M_{0 \rightarrow k}^{-1}$. For consistency with Proposition 7.2, the local behavior of this solution near the singular points is required to be given by

$$
\Phi^{[k]}(z)= \begin{cases}S_{k-1}(-z)^{\mathfrak{S}_{k-1}} G_{0}^{[k]}(z), & z \rightarrow 0, \\ S_{k}(-z)^{\mathfrak{S}_{k}} G_{\infty}^{[k]}(z), & z \rightarrow \infty\end{cases}
$$

where $G_{0}^{[k]}(z), G_{\infty}^{[k]}(z)$ are holomorphic and invertible in the respective neighborhoods of 0 and $\infty$. Using Lemma 4.1, we may fix

$$
\Phi^{[k]}(z)=S_{k-1} \Phi^{(0)}\left(\frac{z}{z_{k}}\right)=S_{k} \Phi^{(\infty)}\left(\frac{z}{z_{k}}\right),
$$

where the parameters of the lemma are identified as $\Theta_{0} \mapsto \mathfrak{S}_{k-1}, \Theta_{\infty} \mapsto-\mathfrak{S}_{k}$. Furthermore, using the relation between $\Phi^{(0)}\left(\frac{z}{z_{k}}\right)$ and $\Phi^{(\infty)}\left(\frac{z}{z_{k}}\right)$ from the same lemma together with the parametrization of Proposition 7.2, we obtain

$$
S_{k-1}^{-1} S_{k}=D_{k-1} W_{k}=\bar{R}^{[k]} S_{\mathfrak{S}_{k-1},-\mathfrak{S}_{k}}\left(R^{[k]}\right)^{-1}
$$

The latter relation connects the diagonal matrices $R^{[k]}$ and $\bar{R}^{[k]}$ which appear in Lemma 4.1 to the parameters of $D_{k}$ in Proposition 7.2:

$$
\bar{r}_{m}^{[k+1]}=\mathrm{e}^{\beta_{k}^{(m)}} \frac{\prod_{l} \Gamma\left(\sigma_{k}^{(m)}-\sigma_{k+1}^{(l)}\right)}{\prod_{l \neq m} \Gamma\left(1+\sigma_{k}^{(m)}-\sigma_{k}^{(l)}\right)}, \quad r_{j}^{[k]}=\frac{\prod_{l} \Gamma\left(\sigma_{k-1}^{(l)}-\sigma_{k}^{(j)}\right)}{\prod_{l \neq j} \Gamma\left(1-\sigma_{k}^{(j)}+\sigma_{k}^{(l)}\right)} .
$$

The final formulas for $\Psi_{\mathcal{C}, \pm}(z)$ are then given by

$$
\begin{aligned}
& \Psi_{\mathcal{C}_{k,(-)}}(z)=R^{[k]} z_{k}^{-\mathfrak{S}_{k}} \Psi_{-\mathfrak{S}_{k}, \mathfrak{S}_{k-1}}\left(\frac{z_{k}}{z}\right)\left(R^{[k]}\right)^{-1} \\
& \Psi_{\mathcal{C}_{k-1,(-)^{k}}}(z)=\bar{R}^{[k]} z_{k}^{-\mathfrak{S}_{k-1}} \Psi_{\mathfrak{S}_{k-1},-\mathfrak{S}_{k}}\left(\frac{z}{z_{k}}\right) G_{-\mathfrak{S}_{k}, \mathfrak{S}_{k-1}}\left(R^{[k]}\right)^{-1}
\end{aligned}
$$

where $\Psi_{\mathfrak{S}, \mathfrak{S}^{\prime}}(z), G_{\mathfrak{S}_{,} \mathfrak{S}^{\prime}}, R^{[k]}$ and $\bar{R}^{[k]}$ are defined by (4.7), (4.5) and (7.7). In combination with (7.3)-(7.6), these formulas make the Fredholm determinant representation (7.2) completely explicit for the tau functions of semi-degenerate Fuchsian systems.

The series representation for $\tau(\boldsymbol{z})$ is obtained similarly to the 4-point case: it suffices to rewrite the operators $A_{ \pm \mp}$ in the Fourier basis and expand the Fredholm determinant into a sum of the principal minors. The structure of (7.3) implies that these minors factorize into products of smaller ones associated to different $\mathcal{T}^{[k]}$, which is a consequence of the linear pants decomposition that we are using. The minors are labeled by $n-3 N$-tuples of Maya diagrams $\left(-\mathbf{q}_{k}, \mathbf{p}_{k}\right)$ associated to each circle $\mathcal{C}_{k}(k=1, \ldots, n-3)$. Furthermore, they are non-zero only 
if each of the $N$-tuples satisfies the condition of global balance $\left|\mathbf{p}_{k}\right|=\left|\mathbf{q}_{k}\right|$. Therefore, the determinant in (7.2) can be represented as

$$
\begin{aligned}
& \operatorname{det}\left(\begin{array}{cc}
\mathbb{1} & \mathrm{A}_{+-} \\
\mathrm{A}_{-+} & \mathbb{1}
\end{array}\right)=\left[Z\left(\mathcal{T}^{[n-2]}\right) \cdots Z\left(\mathcal{T}^{[2]}\right) Z\left(\mathcal{T}^{[1]}\right)\right]_{\varnothing, \varnothing}^{\varnothing, \varnothing} \\
& \quad=\sum_{\substack{\mathbf{p}_{1}, \mathbf{q}_{1}, \ldots, \mathbf{p}_{n-3}, \mathbf{q}_{n-3} \\
\left|\mathbf{p}_{1}\right|=\left|\mathbf{q}_{1}\right|, \ldots,\left|\mathbf{p}_{n-3}\right|=\left|\mathbf{q}_{n-3}\right|}} Z_{\varnothing, \varnothing}^{\mathbf{p}_{n-3}, \mathbf{q}_{n-3}}\left(\mathcal{T}^{[n-2]}\right) \cdots Z_{\mathbf{p}_{2}, \mathbf{q}_{2}}^{\mathbf{p}_{1}, \mathbf{q}_{1}}\left(\mathcal{T}^{[2]}\right) Z_{\mathbf{p}_{1}, \mathbf{q}_{1}}^{\varnothing, \varnothing}\left(\mathcal{T}^{[1]}\right),
\end{aligned}
$$

where, e.g., for pants $\mathcal{T}^{[l]}$ with $l=2 k+1$ we may set

$$
Z_{\mathbf{p}_{l}, \mathbf{q}_{l}}^{\mathbf{p}_{l-1}, \mathbf{q}_{l-1}}\left(\mathcal{T}^{[l]}\right)=\operatorname{det}\left(\begin{array}{cc}
\mathbf{p}_{\mathcal{C}_{l-1},-; \mathcal{C}_{l},+} & -\mathbf{q}_{l-1} \\
\mathrm{a}_{\mathcal{C}_{l-1},-; \mathcal{C}_{l-1},+} \\
\mathrm{a}_{\mathcal{C}_{l},-; \mathcal{C}_{l},+} & \mathrm{a}_{\mathcal{C}_{l},-; \mathcal{C}_{l-1},+}
\end{array}\right) \mathbf{p}_{-\mathbf{q}_{l-1}}^{\mathbf{p}_{l}} .
$$

The above notation means that the determinant is composed of matrix elements from columns $\mathbf{p}_{l},-\mathbf{q}_{l-1}$ and rows $\mathbf{p}_{l-1},-\mathbf{q}_{l}$ of a's written in the Fourier basis:

$$
\begin{array}{ll}
\mathrm{a}_{\mathcal{C}_{l},-; \mathcal{C}_{l},+}\left(z, z^{\prime}\right)=\sum_{p, q \in \mathbb{Z}_{+}^{\prime}} \mathrm{a}_{\mathcal{C}_{l},-q ; \mathcal{C}_{l}, p} z^{-q-\frac{1}{2}} z^{\prime-p-\frac{1}{2}}, & z, z^{\prime} \in \mathcal{C}_{l}, \\
\mathrm{a}_{\mathcal{C}_{l},-; \mathcal{C}_{l-1},+}\left(z, z^{\prime}\right)=\sum_{p, q \in \mathbb{Z}_{+}^{\prime}} \mathrm{a}_{\mathcal{C}_{l},-q ; \mathcal{C}_{l-1},-p} z^{-q-\frac{1}{2}} z^{p-\frac{1}{2}}, & z \in \mathcal{C}_{l}, \quad z^{\prime} \in \mathcal{C}_{l-1}, \\
\mathrm{a}_{\mathcal{C}_{l-1},-; \mathcal{C}_{l},+}\left(z, z^{\prime}\right)=\sum_{p, q \in \mathbb{Z}_{+}^{\prime}} \mathrm{a}_{\mathcal{C}_{l-1}, p ; \mathcal{C}_{l}, q} z^{p-\frac{1}{2}} z^{\prime-q-\frac{1}{2}}, & z \in \mathcal{C}_{l-1}, \quad z^{\prime} \in \mathcal{C}_{l}, \\
\mathrm{a}_{\mathcal{C}_{l-1},-; \mathcal{C}_{l-1},+}\left(z, z^{\prime}\right)=\sum_{p, q \in \mathbb{Z}_{+}^{\prime}} \mathrm{a}_{\mathcal{C}_{l-1}, p ; \mathcal{C}_{l-1},-q} z^{p-\frac{1}{2}} z^{\prime-\frac{1}{2}}, & z, z^{\prime} \in \mathcal{C}_{l-1} .
\end{array}
$$

Equivalents of the matrix elements appearing in the decompositions (7.10a) and (7.10d) were effectively calculated in the 4-point case:

$$
\begin{gathered}
\mathrm{a}_{\mathcal{C}_{l},-q, j ; \mathcal{C}_{l}, p, k}=-\frac{r_{j}^{[l]}}{r_{k}^{[l]}} \frac{\varphi_{-q}^{(j)}\left(\mathfrak{S}_{l-1},-\mathfrak{S}_{l}\right) \bar{\varphi}_{p}^{(k)}\left(\mathfrak{S}_{l-1},-\mathfrak{S}_{l}\right) z_{l}^{q-\sigma_{l}^{(j)}+p+\sigma_{l}^{(k)}}}{q-\sigma_{l}^{(j)}+p+\sigma_{l}^{(k)}}, \\
\mathrm{a}_{\mathcal{C}_{l-1}, p, j ; \mathcal{C}_{l-1},-q, k}=-\frac{\bar{r}_{j}^{[l]}}{\bar{r}_{k}^{[l]}} \frac{\varphi_{-p}^{(j)}\left(-\mathfrak{S}_{l}, \mathfrak{S}_{l-1}\right) \bar{\varphi}_{q}^{(k)}\left(-\mathfrak{S}_{l}, \mathfrak{S}_{l-1}\right) z_{l}^{-p-\sigma_{l-1}^{(j)}-q+\sigma_{l-1}^{(k)}}}{p+\sigma_{l-1}^{(j)}+q-\sigma_{l-1}^{(k)}} .
\end{gathered}
$$

Here $\varphi_{-q}^{(j)}\left(\mathfrak{S},-\mathfrak{S}^{\prime}\right), \bar{\varphi}_{p}^{(k)}\left(\mathfrak{S},-\mathfrak{S}^{\prime}\right)$ are defined by $(5.2 \mathrm{~b})$. The matrix elements in $(7.10 \mathrm{~b})$ and (7.10c) may be obtained by a similar procedure:

$$
\begin{aligned}
& \mathrm{a}_{\mathcal{C}_{l-1}, p, j ; \mathcal{C}_{l}, q, k}=\frac{\bar{r}_{j}^{[l]}}{r_{k}^{[l]}} \frac{\varphi_{-p}^{(j)}\left(-\mathfrak{S}_{l}, \mathfrak{S}_{l-1}\right) \bar{\varphi}_{q}^{(k)}\left(\mathfrak{S}_{l-1},-\mathfrak{S}_{l}\right) z_{l}^{-p-\sigma_{l-1}^{(j)}+q+\sigma_{l}^{(k)}}}{p+\sigma_{l-1}^{(j)}-q-\sigma_{l}^{(k)}}, \\
& \mathrm{a}_{\mathcal{C}_{l},-q, j ; \mathcal{C}_{l-1},-p, k}=\frac{r_{j}^{[l]}}{\bar{r}_{k}^{[l]}} \frac{\varphi_{-q}^{(j)}\left(\mathfrak{S}_{l-1},-\mathfrak{S}_{l}\right) \bar{\varphi}_{p}^{(k)}\left(-\mathfrak{S}_{l}, \mathfrak{S}_{l-1}\right) z_{l}^{q-\sigma_{l}^{(j)}-p+\sigma_{l-1}^{(k)}}}{q-\sigma_{l}^{(j)}-p+\sigma_{l-1}^{(k)}} .
\end{aligned}
$$

Thus, up to an overall sign, the contributions $Z_{\mathbf{p}_{l}, \mathbf{q}_{l}}^{\mathbf{p}_{l-1}, \mathbf{q}_{l-1}}\left(\mathcal{T}^{[l]}\right)$ of individual pairs of pants in (7.9) are given by Cauchy-type determinants:

$$
Z_{\mathbf{p}_{l}, \mathbf{q}_{l}}^{\mathbf{p}_{l-1}, \mathbf{q}_{l-1}}\left(\mathcal{T}^{[l]}\right)= \pm z_{l} \boldsymbol{\sigma}_{l} \cdot \boldsymbol{w}_{l}-\boldsymbol{\sigma}_{l-1} \cdot \boldsymbol{w}_{l-1}+\sum_{q \in \mathbf{q}_{l}} q+\sum_{p \in \mathbf{p}_{l}} p-\sum_{q \in \mathbf{q}_{l-1}} q-\sum_{p \in \mathbf{p}_{l-1}} p
$$




$$
\times \prod_{k=1}^{N}\left(\bar{r}_{k}^{[l]}\right)^{w_{l-1}^{(k)}}\left(r_{k}^{[l]}\right)^{-w_{l}^{(k)}} Z_{\mathbf{p}_{l}, \mathbf{q}_{l}}^{\mathbf{p}_{l-1}, \mathbf{q}_{l-1}}\left(\mathfrak{S}_{l-1}, \mathfrak{S}_{l}\right)
$$

where

$$
\begin{aligned}
Z_{\mathbf{p}, \mathbf{q}}^{\mathbf{p}^{\prime}, \mathbf{q}^{\prime}}\left(\mathfrak{S}^{\prime}, \mathfrak{S}\right)= & \prod_{(p, j) \in \mathbf{p}^{\prime}} \varphi_{-p}^{(j)}\left(-\mathfrak{S}, \mathfrak{S}^{\prime}\right) \prod_{(q, k) \in \mathbf{q}^{\prime}} \bar{\varphi}_{q}^{(k)}\left(-\mathfrak{S}, \mathfrak{S}^{\prime}\right) \\
& \times \prod_{(q, j) \in \mathbf{q}} \varphi_{-q}^{(j)}\left(\mathfrak{S}^{\prime},-\mathfrak{S}\right) \prod_{(p, k) \in \mathbf{p}} \bar{\varphi}_{p}^{(k)}\left(\mathfrak{S}^{\prime},-\mathfrak{S}\right) \\
& \times \operatorname{det}\left(\frac{\mathbf{p}}{\frac{1}{p^{\prime}+\sigma^{\prime(j)}-p-\sigma^{(k)}}} \frac{-\mathbf{q}^{\prime}}{\frac{1}{-q+\sigma^{(j)}-p-\sigma^{(k)}}} \frac{1}{-q+\sigma^{(j)}+q^{\prime}-\sigma^{(k)}}\right)_{-\mathbf{q}}^{\prime}
\end{aligned}
$$

and $\boldsymbol{w}_{l} \in \mathfrak{Q}_{N-1}$ denotes the charge vector of the $N$-tuple of Maya diagrams assigned to the circle $\mathcal{C}_{l}$. Substituting the expressions (7.13) into the principal minor expansion, we obtain a multivariate analog of the series representation (5.5) of the $\mathrm{FST}_{N}$ tau function. The result is expressed in terms of $\boldsymbol{\sigma}_{1}, \ldots, \boldsymbol{\sigma}_{n-3}$ and $\boldsymbol{\beta}_{1}, \ldots, \boldsymbol{\beta}_{n-3}$, the latter parameters entering only via (7.7).

\section{A AGT-W relation from isomonodromy}

We start with a brief review of semi-degenerate vertex operators of $W_{N}$-algebras and the corresponding conformal blocks (CBs), trying to highlight the general structures without entering into the details. The $W_{N}$-algebras are infinite-dimensional associative algebras generalizing Virasoro algebra which is $W_{2}$-algebra. The Verma modules of $W_{N}=W\left(\mathfrak{s l}_{N}\right)$ are labeled by the weights $\boldsymbol{\sigma}=\left(\sigma^{(1)}, \ldots, \sigma^{(N)}\right) \in \mathbb{C}^{N}, \sum_{\alpha=1}^{N} \sigma^{(\alpha)}=0$, of the Lie algebra $\mathfrak{s l}_{N}$. These Verma modules are irreducible for generic $\boldsymbol{\sigma}$. We denote by $\mathcal{V}_{\boldsymbol{\sigma}}$ the irreducible Verma modules or irreducible quotients with the same highest weight vectors $|\boldsymbol{\sigma}\rangle$ of reducible Verma modules. Important examples of irreducible quotients are semi-degenerate $W_{N}$-modules $\mathcal{V}_{\boldsymbol{\sigma}}$ with $\boldsymbol{\sigma}=\Lambda \boldsymbol{h}_{1}$, where $\Lambda \in \mathbb{C}$ and $\boldsymbol{h}_{1}=\left(\frac{N-1}{N},-\frac{1}{N}, \ldots,-\frac{1}{N}\right)$ is the 1 st fundamental weight of $\mathfrak{s l}_{N}$.

In order to define CBs of the $W_{N}$-algebra on $\mathbb{C P}^{1}$, one has to fix $n$ points $\boldsymbol{z}:=\left\{z_{0} \equiv 0, z_{1}, \ldots\right.$, $\left.z_{n-2}, z_{n-1} \equiv \infty\right\}$ and attach a $W_{N}$-module $\mathcal{V}_{\boldsymbol{\theta}_{k}}$ to each of them. The $n$-point CBs are linear forms on $\mathcal{V}_{\boldsymbol{\theta}_{0}} \otimes \cdots \otimes \mathcal{V}_{\boldsymbol{\theta}_{n-1}}$ satisfying the so-called conformal Ward identities. In general, the space of such CBs is infinite-dimensional. For its description, it is useful to decompose $\mathbb{C P}^{1} \backslash \boldsymbol{z}$ into pairs of pants as above. With each pair of pants $\mathcal{T}^{[k]}(k=1, \ldots, n-2)$ is associated a space of 3-point CBs if we fix $W_{N}$-modules $\mathcal{V}_{\sigma_{l}}$ on cutting circles $\mathcal{C}_{l}(l=1, \ldots, n-3)$. Such 3-point CBs are linear forms on $\mathcal{V}_{\boldsymbol{\sigma}_{k}} \otimes \mathcal{V}_{\boldsymbol{\theta}_{k}} \otimes \mathcal{V}_{\boldsymbol{\sigma}_{k-1}}$, where $\boldsymbol{\sigma}_{0}=\boldsymbol{\theta}_{0}$ and $\boldsymbol{\sigma}_{n-2}=-\boldsymbol{\theta}_{n-1}$. For generic $\boldsymbol{\sigma}_{k}$, $\boldsymbol{\theta}_{k}, \boldsymbol{\sigma}_{k-1}$, this space of 3 -point CBs is also infinite-dimensional. However, in certain special cases, e.g., for semi-degenerate $\mathcal{V}_{\boldsymbol{\theta}_{k}}$ (with $\boldsymbol{\theta}_{k}=\Lambda_{k} \boldsymbol{h}_{1}$ ), it becomes one-dimensional, similarly to the space of generic 3-point Virasoro CBs.

Using the Shapovalov non-degenerate bilinear form on irreducible modules $\mathcal{V}_{\boldsymbol{\sigma}}$, one can rewrite linear forms on $\mathcal{V}_{\boldsymbol{\sigma}_{k}} \otimes\left|\boldsymbol{\theta}_{k}\right\rangle \otimes \mathcal{V}_{\boldsymbol{\sigma}_{k-1}}$, where $\left|\boldsymbol{\theta}_{k}\right\rangle$ is highest weight vector of $\mathcal{V}_{\boldsymbol{\theta}_{k}}$, as linear operators $V_{\boldsymbol{\theta}_{k}}\left(z_{k}\right): \mathcal{V}_{\boldsymbol{\sigma}_{k-1}} \rightarrow \mathcal{V}_{\boldsymbol{\sigma}_{k}}$, which are called vertex operators. In the case of semi-degenerate $\boldsymbol{\theta}_{k}=$ $\Lambda_{k} \boldsymbol{h}_{1}$, the space of such operators is one-dimensional, and they are uniquely fixed by their matrix elements between the highest weight vectors. E.g., for the central charge $c=N-1$, they read

$$
\left\langle\boldsymbol{\sigma}_{k}\left|V_{\Lambda_{k}}\left(z_{k}\right)\right| \boldsymbol{\sigma}_{k-1}\right\rangle=\mathcal{N}\left(\boldsymbol{\sigma}_{k}, \Lambda_{k} \boldsymbol{h}_{1}, \boldsymbol{\sigma}_{k-1}\right) z_{k}^{\frac{1}{2}\left(\boldsymbol{\sigma}_{k}^{2}-\Lambda_{k}^{2} \boldsymbol{h}_{1}^{2}-\boldsymbol{\sigma}_{k-1}^{2}\right)},
$$


where we used a shorthand notation $V_{\Lambda_{k}}\left(z_{k}\right)$ for the corresponding semi-degenerate vertex operator.

In [1], a relation between instanton partition functions and Virasoro CBs was conjectured. Later in [32], this proposal was extended to semi-degenerate CBs of $W_{N}$-algebras. The conjectured relation is as follows [10]: there exist bases of $W_{N}$-modules $\mathcal{V}_{\boldsymbol{\sigma}}$ labeled by $N$-tuples of Young diagrams $\boldsymbol{Y}=\left(Y^{(1)}, \ldots, Y^{(N)}\right) \in \mathbb{Y}^{N}$ such that the matrix elements of semi-degenerate vertex operators are given by (for simplicity, we again restrict ourselves to $c=N-1$ which is the only case relevant to our purposes)

$$
F_{\boldsymbol{Y}, \boldsymbol{Y}^{\prime}}\left(\boldsymbol{\sigma}, \Lambda, \boldsymbol{\sigma}^{\prime}\right):=\frac{\left\langle\boldsymbol{\sigma}, \boldsymbol{Y}\left|V_{\Lambda}(1)\right| \boldsymbol{\sigma}^{\prime}, \boldsymbol{Y}^{\prime}\right\rangle}{\left\langle\boldsymbol{\sigma}\left|V_{\Lambda}(1)\right| \boldsymbol{\sigma}^{\prime}\right\rangle}=\prod_{\alpha, \beta=1}^{N} Z_{\mathrm{bif}}\left(\sigma^{(\alpha)}-\sigma^{(\beta)}-\frac{\Lambda}{N} \mid Y^{\prime(\alpha)}, Y^{(\beta)}\right),
$$

where

$$
Z_{\text {bif }}\left(\nu \mid Y^{\prime}, Y\right):=\prod_{\square \in Y^{\prime}}\left(\nu+1+a_{Y^{\prime}}(\square)+l_{Y}(\square)\right) \prod_{\square \in Y}\left(\nu-1-a_{Y}(\square)-l_{Y^{\prime}}(\square)\right),
$$

and $a_{Y}(\square), l_{Y}(\square)$ denote the arm-length and leg-length of the box $\square$ with respect to $Y$. The basis $\{|\boldsymbol{\sigma}, \boldsymbol{Y}\rangle\}_{\boldsymbol{Y} \in \mathbb{Y}^{N}}$ is orthogonal with respect to the Shapovalov form:

$$
\left\langle\boldsymbol{\sigma}, \boldsymbol{Y} \mid \boldsymbol{\sigma}^{\prime}, \boldsymbol{Y}^{\prime}\right\rangle=\delta_{\boldsymbol{\sigma}, \boldsymbol{\sigma}^{\prime}} \delta_{\boldsymbol{Y}, \boldsymbol{Y}^{\prime}} F_{\boldsymbol{Y}, \boldsymbol{Y}}(\boldsymbol{\sigma}, 0, \boldsymbol{\sigma}),
$$

corresponding to the matrix elements of the identity vertex operator $V_{\mathbf{0}}(1)$. Therefore, the projection $\mathcal{P}_{\boldsymbol{\sigma}}$ to the $W_{N}$-module $\mathcal{V}_{\boldsymbol{\sigma}}$ is

$$
\mathcal{P}_{\boldsymbol{\sigma}}=\sum_{\boldsymbol{Y}} \frac{|\boldsymbol{\sigma}, \boldsymbol{Y}\rangle\langle\boldsymbol{\sigma}, \boldsymbol{Y}|}{\langle\boldsymbol{\sigma}, \boldsymbol{Y} \mid \boldsymbol{\sigma}, \boldsymbol{Y}\rangle}
$$

Combining these ingredients together, one comes to combinatorial expressions for semi-degenerate CBs. For instance, the 4 -point $\mathrm{CBs}$ are given by

$$
\begin{aligned}
& \frac{\left\langle-\boldsymbol{\theta}_{\infty}\left|V_{\Lambda_{3}}(1) \mathcal{P}_{\boldsymbol{\sigma}} V_{\Lambda_{2}}(z)\right| \boldsymbol{\theta}_{0}\right\rangle}{\left\langle-\boldsymbol{\theta}_{\infty}\left|V_{\Lambda_{3}}(1)\right| \boldsymbol{\sigma}\right\rangle\left\langle\boldsymbol{\sigma}\left|V_{\Lambda_{2}}(1)\right| \boldsymbol{\theta}_{0}\right\rangle}=(1-z)^{-\frac{\Lambda_{3} \Lambda_{2}}{N}} z^{\frac{1}{2}\left(\boldsymbol{\sigma}^{2}-\Lambda_{2}^{2} \boldsymbol{h}_{1}^{2}-\boldsymbol{\theta}_{0}^{2}\right)} \\
& \times \sum_{\boldsymbol{Y} \in \mathbb{Y}^{N}} \frac{F_{\varnothing, \boldsymbol{Y}}\left(-\boldsymbol{\theta}_{\infty}, \Lambda_{3}, \boldsymbol{\sigma}\right) F_{\boldsymbol{Y}, \varnothing}\left(\boldsymbol{\sigma}, \Lambda_{2}, \boldsymbol{\theta}_{0}\right)}{F_{\boldsymbol{Y}, \boldsymbol{Y}}(\boldsymbol{\sigma}, 0, \boldsymbol{\sigma})} z^{|\boldsymbol{Y}|} .
\end{aligned}
$$

As was shown in our previous work [14] (generalizing the connection of Painlevé VI and Garnier system to Liouville CFT $[2,13,17])$, the tau function $\tau(\boldsymbol{z})$ of the $n$-point semi-degenerate Fuchsian system can be represented as a Fourier transform of $n$-point semi-degenerate $W_{N}$ conformal blocks.

Theorem A.1. The tau function $\tau(\boldsymbol{z})$ can be expressed in terms of semi-degenerate $W_{N}$ conformal blocks with central charge $c=N-1$ as

$$
\begin{aligned}
& \tau(\boldsymbol{z})=\sum_{\boldsymbol{w}_{1}, \ldots, \boldsymbol{w}_{n-3} \in \mathfrak{Q}_{N-1}} \mathrm{e}^{\boldsymbol{\beta}_{1} \cdot \boldsymbol{w}_{1}+\cdots+\boldsymbol{\beta}_{n-3} \cdot \boldsymbol{w}_{n-3}} \mathcal{F}\left(\boldsymbol{\sigma}_{1}+\boldsymbol{w}_{1}, \ldots, \boldsymbol{\sigma}_{n-3}+\boldsymbol{w}_{n-3}\right), \\
& \mathcal{F}\left(\boldsymbol{\sigma}_{1}, \ldots, \boldsymbol{\sigma}_{n-3}\right)=\left\langle-\boldsymbol{\theta}_{n-1}\left|V_{\Lambda_{n-2}}\left(z_{n-2}\right) \mathcal{P}_{\boldsymbol{\sigma}_{n-3}} V_{\Lambda_{n-3}}\left(z_{n-3}\right) \mathcal{P}_{\boldsymbol{\sigma}_{n-4}} \cdots \mathcal{P}_{\boldsymbol{\sigma}_{1}} V_{\Lambda_{1}}\left(z_{1}\right)\right| \boldsymbol{\theta}_{0}\right\rangle,
\end{aligned}
$$

where $\left(\boldsymbol{\sigma}_{1}, \ldots, \boldsymbol{\sigma}_{n-3}\right)$ and $\left(\boldsymbol{\beta}_{1}, \ldots, \boldsymbol{\beta}_{n-3}\right)$ are parameters fixing the initial conditions, directly related to monodromy parameterization in Proposition 7.2 :

$$
\mathfrak{S}_{l} \rightarrow \operatorname{diag}\left\{\boldsymbol{\sigma}_{l}\right\}+\frac{\sum_{j=1}^{l} \Lambda_{j}}{N} \mathbb{1}, \quad D_{l}=\operatorname{diag}\left\{\mathrm{e}^{\beta_{l}^{(1)}}, \ldots, \mathrm{e}^{\beta_{l}^{(N)}}\right\} .
$$


The vertex operators $V_{\Lambda}(z)$ are normalized by (A.1) with

$$
\mathcal{N}\left(\boldsymbol{\sigma}, \Lambda \boldsymbol{h}_{1}, \boldsymbol{\sigma}^{\prime}\right)=\frac{\prod_{l, j} G\left(1+\sigma^{\prime(l)}-\sigma^{(j)}-\frac{\Lambda}{N}\right)}{\prod_{k<m} G\left(1+\sigma^{\prime(k)}-\sigma^{\prime(m)}\right) G\left(1-\sigma^{(k)}+\sigma^{(m)}\right)},
$$

where $G(x)$ denotes the Barnes G-function.

We would like to compare this expression for $\tau(\boldsymbol{z})$ with the one coming from (7.2) - namely, with the combinatorial expansion (7.8) in terms of Cauchy determinants (7.13). It will be shown that the two expansions coincide termwise. Besides the check of consistency of the two approaches, this yields as a byproduct a direct proof of Nekrasov/AGT-W formulas for semidegenerate conformal blocks of the $W_{N}$ algebra with $c=N-1$. We are going to proceed along the lines of [15, Appendix A], which deals with the $N=2$ case but contains several useful results independent of $N$.

We will need an extension of the formula (A.3) for charged Young diagrams $Y, Y^{\prime} \in \mathbb{Y}$ with charges $w, w^{\prime} \in \mathbb{Z}$ :

$$
\begin{aligned}
\tilde{Z}_{\mathrm{bif}}\left(\nu \mid w^{\prime}, Y^{\prime} ; w, Y\right)= & \prod_{i}(-\nu)_{q_{i}^{\prime}+\frac{1}{2}} \prod_{i}(\nu+1)_{q_{i}-\frac{1}{2}} \prod_{i}(-\nu)_{p_{i}+\frac{1}{2}} \prod_{i}(\nu+1)_{p_{i}^{\prime}-\frac{1}{2}} \\
& \times \frac{\prod_{i, j}\left(\nu-q_{i}^{\prime}-p_{j}\right) \prod_{i, j}\left(\nu+p_{i}^{\prime}+q_{j}\right)}{\prod_{i, j}\left(\nu-q_{i}^{\prime}+q_{j}\right) \prod_{i, j}\left(\nu+p_{i}^{\prime}-p_{j}\right)},
\end{aligned}
$$

where we used the Frobenius coordinates $(\mathbf{p}, \mathbf{q})$ of charged Young diagrams $(w, Y)$. It follows from Theorems A.1 and A.5 of [15] that $\tilde{Z}_{\mathrm{bif}}\left(\nu \mid w^{\prime}, Y^{\prime} ; w, Y\right)$ for charged Young diagrams can be reduced to an expression for non-charged ones:

$$
\tilde{Z}_{\mathrm{bif}}\left(\nu \mid w^{\prime}, Y^{\prime} ; w, Y\right)= \pm C\left(\nu \mid w^{\prime}, w\right) Z_{\mathrm{bif}}\left(\nu+w^{\prime}-w \mid Y^{\prime}, Y\right) .
$$

Here

$$
C\left(\nu \mid w^{\prime}, w\right) \equiv C\left(\nu \mid w^{\prime}-w\right)=\frac{G\left(1+\nu+w^{\prime}-w\right)}{G(1+\nu) \Gamma(1+\nu)^{w^{\prime}-w}} .
$$

The only property of $G(x)$ relevant for us is the recurrence relation $G(x+1)=\Gamma(x) G(x)$. In order to lighten the formulas, the calculations below will be carried out only up to an overall sign which can be controlled by considering a suitable limit of parameters, e.g., $\nu \rightarrow+\mathrm{i} \infty$ in (A.6).

Our starting point is the expression (7.14). Rewrite the Cauchy determinant therein in the factorized form and compare $Z_{\mathbf{p}, \mathbf{q}}^{\mathbf{p}^{\prime}, \mathbf{q}^{\prime}}\left(\mathfrak{S}^{\prime}, \mathfrak{S}\right)$ with the following Cauchy-like factorized expression:

$$
\begin{aligned}
& \tilde{Z}_{\boldsymbol{Y}, \boldsymbol{w}}^{\boldsymbol{Y}^{\prime}, \boldsymbol{w}^{\prime}}\left(\mathfrak{S}^{\prime}, \mathfrak{S}\right)=\prod_{j=1}^{N}\left|\tilde{Z}_{\mathrm{bif}}\left(0 \mid w^{(j)}, Y^{(j)} ; w^{(j)}, Y^{(j)}\right)\right|^{-\frac{1}{2}}\left|\tilde{Z}_{\mathrm{bif}}\left(0 \mid w^{\prime(j)}, Y^{\prime(j)} ; w^{\prime(j)}, Y^{\prime(j)}\right)\right|^{-\frac{1}{2}} \\
& \times \frac{\prod_{j, k=1}^{N} \tilde{Z}_{\mathrm{bif}}\left(\sigma^{\prime(j)}-\sigma^{(k)} \mid w^{\prime(j)}, Y^{\prime(j)} ; w^{(k)}, Y^{(k)}\right)}{\prod_{1 \leq j<k \leq N} \tilde{Z}_{\mathrm{bif}}\left(\sigma^{\prime(j)}-\sigma^{\prime(k)} \mid w^{\prime(j)}, Y^{\prime(j)} ; w^{\prime(k)}, Y^{\prime(k)}\right) \tilde{Z}_{\mathrm{bif}}\left(\sigma^{(k)}-\sigma^{(j)} \mid w^{(k)}, Y^{(k)} ; w^{(j)}, Y^{(j)}\right)} .
\end{aligned}
$$

The result of comparison is

$$
Z_{\mathbf{p}, \mathbf{q}}^{\mathbf{p}^{\prime}, \mathbf{q}^{\prime}}\left(\mathfrak{S}^{\prime}, \mathfrak{S}\right)=\tilde{Z}_{\boldsymbol{Y}, \boldsymbol{w}}^{\boldsymbol{Y}^{\prime}, \boldsymbol{w}^{\prime}}\left(\mathfrak{S}^{\prime}, \mathfrak{S}\right) \prod_{(p, j) \in \mathbf{p}^{\prime}} \delta \varphi^{(j)}\left(-\mathfrak{S}, \mathfrak{S}^{\prime}\right)
$$




$$
\times \prod_{(q, k) \in \mathbf{q}^{\prime}} \delta \bar{\varphi}^{(k)}\left(-\mathfrak{S}, \mathfrak{S}^{\prime}\right) \prod_{(q, j) \in \mathbf{q}} \delta \varphi^{(j)}\left(\mathfrak{S}^{\prime},-\mathfrak{S}\right) \prod_{(p, k) \in \mathbf{p}} \delta \bar{\varphi}^{(k)}\left(\mathfrak{S}^{\prime},-\mathfrak{S}\right),
$$

where

$$
\delta \varphi^{(k)}\left(-\mathfrak{S}, \mathfrak{S}^{\prime}\right)=\left(\delta \bar{\varphi}^{(k)}\left(-\mathfrak{S}, \mathfrak{S}^{\prime}\right)\right)^{-1}=\prod_{j=1}^{k-1}\left({\sigma^{\prime}}^{(k)}-{\sigma^{\prime}}^{(j)}\right) \prod_{j=1}^{N}\left(\sigma^{(j)}-\sigma^{\prime(k)}\right) .
$$

Since these expressions are independent of momentum $p$, it follows that

$$
Z_{\mathbf{p}, \mathbf{q}}^{\mathbf{p}^{\prime}, \mathbf{q}^{\prime}}\left(\mathfrak{S}^{\prime}, \mathfrak{S}\right)=\tilde{Z}_{\boldsymbol{Y}, \boldsymbol{w}}^{\boldsymbol{Y}^{\prime}, \boldsymbol{w}^{\prime}}\left(\mathfrak{S}^{\prime}, \mathfrak{S}\right) \cdot \prod_{k=1}^{N}\left(\delta \varphi^{(k)}\left(-\mathfrak{S}, \mathfrak{S}^{\prime}\right)\right)^{w^{\prime(k)}}\left(\delta \varphi^{(k)}\left(\mathfrak{S}^{\prime},-\mathfrak{S}\right)\right)^{-w^{(k)}}
$$

Using (A.6) we can rewrite $\tilde{Z}_{\boldsymbol{Y}, \boldsymbol{w}}^{\boldsymbol{Y}^{\prime}, \boldsymbol{w}^{\prime}}\left(\mathfrak{S}^{\prime}, \mathfrak{S}\right)$ in terms of non-charged Young diagrams:

$$
\begin{aligned}
\tilde{Z}_{\boldsymbol{Y}, \boldsymbol{w}}^{\boldsymbol{Y}^{\prime}, \boldsymbol{w}^{\prime}}\left(\mathfrak{S}^{\prime}, \mathfrak{S}\right)= & \tilde{Z}_{\boldsymbol{Y}}^{\boldsymbol{Y}^{\prime}}\left(\boldsymbol{\sigma}^{\prime}+\boldsymbol{w}^{\prime}, \boldsymbol{\sigma}+\boldsymbol{w}\right) \frac{\mathcal{N}\left(\boldsymbol{\sigma}+\boldsymbol{w}, 0, \boldsymbol{\sigma}^{\prime}+\boldsymbol{w}^{\prime}\right)}{\mathcal{N}\left(\boldsymbol{\sigma}, 0, \boldsymbol{\sigma}^{\prime}\right)} \\
& \times \prod_{k=1}^{N}\left(\delta \Gamma^{(k)}\left(-\mathfrak{S}, \mathfrak{S}^{\prime}\right)\right)^{w^{\prime(k)}}\left(\delta \Gamma^{(k)}\left(\mathfrak{S}^{\prime},-\mathfrak{S}\right)\right)^{-w^{(k)}}
\end{aligned}
$$

Here the structure constants $\mathcal{N}(\cdot)$ are defined in $(\mathrm{A} .5), \tilde{Z}_{\boldsymbol{Y}}^{\boldsymbol{Y}^{\prime}}\left(\boldsymbol{\sigma}^{\prime}, \boldsymbol{\sigma}\right)$ is given by

$$
\begin{aligned}
\tilde{Z}_{\boldsymbol{Y}}^{\boldsymbol{Y}^{\prime}}\left(\boldsymbol{\sigma}^{\prime}, \boldsymbol{\sigma}\right)= & \prod_{j=1}^{N}\left|Z_{\mathrm{bif}}\left(0 \mid Y^{(j)}, Y^{(j)}\right)\right|^{-\frac{1}{2}}\left|Z_{\mathrm{bif}}\left(0 \mid Y^{\prime(j)}, Y^{\prime(j)}\right)\right|^{-\frac{1}{2}} \\
& \times \frac{\prod_{j, k=1}^{N} Z_{\mathrm{bif}}\left(\sigma^{\prime(j)}-\sigma^{(k)} \mid Y^{\prime(j)}, Y^{(k)}\right)}{\prod_{j<k} Z_{\mathrm{bif}}\left(\sigma^{\prime(j)}-\sigma^{\prime(k)} \mid Y^{\prime(j)}, Y^{\prime(k)}\right) Z_{\mathrm{bif}}\left(\sigma^{(k)}-\sigma^{(j)} \mid Y^{(k)}, Y^{(j)}\right)},
\end{aligned}
$$

and

$$
\delta \Gamma^{(k)}\left(-\mathfrak{S}, \mathfrak{S}^{\prime}\right)=\frac{\prod_{j>k} \Gamma\left(1+{\sigma^{\prime}}^{(k)}-{\sigma^{\prime}}^{(j)}\right)}{\prod_{j<k} \Gamma\left(1+\sigma^{\prime(j)}-\sigma^{\prime(k)}\right) \prod_{j} \Gamma\left(1+\sigma^{\prime(k)}-\sigma^{(j)}\right)} .
$$

Observe that the products appearing in the right side of (A.8) and (A.9) have the same structure as the product of $\bar{r}_{k}^{[l]}$ and $r_{k}^{[l]}$ in (7.13). Combining these products together produces the Fourier exponents in (A.4). Indeed, collect the contributions of the form $\left(B_{l}^{(k)}\right)^{w_{l}^{(k)}}$ associated with the circle $\mathcal{C}_{l}$ coming from the different factors in (7.8):

$$
Z_{\mathbf{p}_{l+1}, \mathbf{q}_{l+1}}^{\mathbf{p}_{l}, \mathbf{q}_{l}}\left(\mathcal{T}^{[l+1]}\right) Z_{\mathbf{p}_{l}, \mathbf{q}_{l}, \mathbf{q}_{l-1}}^{\mathbf{p}_{l}}\left(\mathcal{T}^{[l]}\right) \quad \Longrightarrow \quad \prod_{k=1}^{N}\left(B_{l}^{(k)}\right)^{w_{l}^{(k)}}
$$

Using (7.13), (A.7)-(A.11) and then (7.7), we obtain

$$
B_{l}^{(k)}=\frac{\bar{r}_{k}^{[l+1]}}{r_{k}^{[l]}} \cdot \frac{\delta \varphi^{(k)}\left(-\mathfrak{S}_{l+1}, \mathfrak{S}_{l}\right)}{\delta \varphi^{(k)}\left(\mathfrak{S}_{l-1},-\mathfrak{S}_{l}\right)} \cdot \frac{\delta \Gamma^{(k)}\left(-\mathfrak{S}_{l+1}, \mathfrak{S}_{l}\right)}{\delta \Gamma^{(k)}\left(\mathfrak{S}_{l-1},-\mathfrak{S}_{l}\right)}=\mathrm{e}^{\beta_{l}^{(k)}}
$$


The dependence of (7.13) on the positions of semi-degenerate poles is concentrated in the factors

$$
\begin{aligned}
& \boldsymbol{\sigma}_{l} \cdot \boldsymbol{w}_{l}-\boldsymbol{\sigma}_{l-1} \cdot \boldsymbol{w}_{l-1}+\sum_{q \in \mathbf{q}_{l}} q+\sum_{p \in \mathbf{p}_{l}} p-\sum_{q \in \mathbf{q}_{l-1}} q-\sum_{p \in \mathbf{p}_{l-1}} p \\
& z_{l} \\
& =z_{l}^{\frac{1}{2}\left(\left(\boldsymbol{\sigma}_{l}+\boldsymbol{w}_{l}\right)^{2}-\boldsymbol{\sigma}_{l}^{2}-\left(\boldsymbol{\sigma}_{l-1}+\boldsymbol{w}_{l-1}\right)^{2}+\boldsymbol{\sigma}_{l-1}^{2}\right)-\left|\boldsymbol{Y}_{l-1}\right|+\left|\boldsymbol{Y}_{l}\right|},
\end{aligned}
$$

where $|\boldsymbol{Y}|$ denotes the total number of cells in the $N$-tuple $\mathbf{Y} \in \mathbb{Y}^{N}$, cf., e.g., [15, Fig. 13]. Taking into account the product of 3-point tau functions in (7.2), each summand of (7.8) produces the following $\boldsymbol{z}$-dependent contribution to the expansion of $\tau(\boldsymbol{z})$ :

$$
\prod_{l=1}^{n-2} z_{l}^{\frac{1}{2}\left(\left(\boldsymbol{\sigma}_{l}+\boldsymbol{w}_{l}\right)^{2}-\left(\boldsymbol{\sigma}_{l-1}+\boldsymbol{w}_{l-1}\right)^{2}+\Lambda_{l}^{2}\right)-\left|\boldsymbol{Y}_{l-1}\right|+\left|\boldsymbol{Y}_{l}\right|} \text {. }
$$

The remaining factors $\tilde{Z}_{\boldsymbol{Y}}^{Y^{\prime}}\left(\boldsymbol{\sigma}^{\prime}, \boldsymbol{\sigma}\right)$, coming to $Z_{\mathbf{p}, \mathbf{q}^{\mathbf{p}^{\prime}}, \mathbf{q}^{\prime}}\left(\mathfrak{S}^{\prime}, \mathfrak{S}\right)$ from $\tilde{Z}_{\boldsymbol{Y}, \boldsymbol{w}}^{\boldsymbol{Y}^{\prime}, \boldsymbol{w}^{\prime}}\left(\mathfrak{S}^{\prime}, \mathfrak{S}\right)$, reproduce the gauge theory prediction (A.2) for the matrix elements of semi-degenerate vertex operators. To identify the corresponding formulas, one should take into account that Cauchy matrices (7.11)(7.12) arise when $\operatorname{rk} A_{1}=\cdots=\operatorname{rk} A_{n-2}=1$ in (7.1). The transformation to the "CFT gauge" (where all $\operatorname{Tr} A_{k}=0$ ) shifts $\mathfrak{S}_{k}$ 's by appropriate scalars, which effectively leads to $\frac{\Lambda}{N}$-translations in the formulas such as (A.2) and (A.5).

\section{Acknowledgements}

The work of P.G. was partially supported by the Russian Academic Excellence Project '5-100' and by the RSF grant No. 16-11-10160. In particular, the results of Section 6 were obtained using support of Russian Science Foundation. P.G. is a Young Russian Mathematics award winner and would like to thank its sponsors and jury. N.I. was partially supported by the National Academy of Sciences of Ukraine (project No. 0117U000238), by the Program of Fundamental Research of the Department of Physics and Astronomy of the NAS of Ukraine (project No. 0117U000240), and by the ICTP-SEENET-MTP project NT-03: Cosmology - Classical and Quantum Challenges.

\section{References}

[1] Alday L.F., Gaiotto D., Tachikawa Y., Liouville correlation functions from four-dimensional gauge theories, Lett. Math. Phys. 91 (2010), 167-197, arXiv:0906.3219.

[2] Bershtein M.A., Shchechkin A.I., Bilinear equations on Painlevé $\tau$ functions from CFT, Comm. Math. Phys. 339 (2015), 1021-1061, arXiv:1406.3008.

[3] Borodin A., Deift P., Fredholm determinants, Jimbo-Miwa-Ueno $\tau$-functions, and representation theory, Comm. Pure Appl. Math. 55 (2002), 1160-1230, math-ph/0111007.

[4] Borodin A., Olshanski G., Harmonic analysis on the infinite-dimensional unitary group and determinantal point processes, Ann. of Math. 161 (2005), 1319-1422, math.RT/0109194.

[5] Cafasso M., Gavrylenko P., Lisovyy O., Tau functions as Widom constants, Comm. Math. Phys., to appear, arXiv:1712.08546.

[6] Chekhov L., Mazzocco M., Isomonodromic deformations and twisted Yangians arising in Teichmüller theory, Adv. Math. 226 (2011), 4731-4775, arXiv:0909.5350.

[7] Deift P., Its A., Kapaev A., Zhou X., On the algebro-geometric integration of the Schlesinger equations, Comm. Math. Phys. 203 (1999), 613-633.

[8] Dubrovin B., Geometry of 2D topological field theories, in Integrable Systems and Quantum Groups (Montecatini Terme, 1993), Lecture Notes in Math., Vol. 1620, Springer, Berlin, 1996, 120-348, hep-th/9407018. 
[9] Dubrovin B., Mazzocco M., Monodromy of certain Painlevé-VI transcendents and reflection groups, Invent. Math. 141 (2000), 55-147, math.AG/9806056.

[10] Fateev V.A., Litvinov A.V., Integrable structure, W-symmetry and AGT relation, J. High Energy Phys. 2012 (2012), no. 1, 051, 39 pages, arXiv:1109.4042.

[11] Fokas A.S., Its A.R., Kapaev A.A., Novokshenov V.Yu., Painlevé transcendents: the Riemann-Hilbert approach, Mathematical Surveys and Monographs, Vol. 128, Amer. Math. Soc., Providence, RI, 2006.

[12] Fuji K., Suzuki T., Drinfeld-Sokolov hierarchies of type $A$ and fourth order Painlevé systems, Funkcial. Ekvac. 53 (2010), 143-167, arXiv:0904.3434.

[13] Gamayun O., Iorgov N., Lisovyy O., Conformal field theory of Painlevé VI, J. High Energy Phys. 2012 (2012), no. 10, 038, 25 pages, arXiv:1207.0787.

[14] Gavrylenko P., Iorgov N., Lisovyy O., Higher rank isomonodromic deformations and $W$-algebras, arXiv:1801.09608.

[15] Gavrylenko P., Lisovyy O., Fredholm determinant and Nekrasov sum representations of isomonodromic tau functions, Comm. Math. Phys. 363 (2018), 1-58, arXiv:1608.00958.

[16] Gavrylenko P., Lisovyy O., Pure SU(2) gauge theory partition function and generalized Bessel kernel, in String-Math 2016, Proc. Sympos. Pure Math., Vol. 98, Amer. Math. Soc., Providence, RI, 2018, 181-205, arXiv:1705.01869.

[17] Iorgov N., Lisovyy O., Teschner J., Isomonodromic tau-functions from Liouville conformal blocks, Comm. Math. Phys. 336 (2015), 671-694, arXiv:1401.6104.

[18] Jimbo M., Miwa T., Ueno K., Monodromy preserving deformation of linear ordinary differential equations with rational coefficients. I. General theory and $\tau$-function, Phys. D 2 (1981), 306-352.

[19] Kitaev A.V., Korotkin D.A., On solutions of the Schlesinger equations in terms of $\Theta$-functions, Int. Math. Res. Not. 1998 (1998), 877-905, math-ph/9810007.

[20] Korotkin D.A., Solution of matrix Riemann-Hilbert problems with quasi-permutation monodromy matrices, Math. Ann. 329 (2004), 335-364, math-ph/0306061.

[21] Lisovyy O., Tykhyy Y., Algebraic solutions of the sixth Painlevé equation, J. Geom. Phys. 85 (2014), 124-163, arXiv:0809.4873.

[22] Mano T., Tsuda T., Hermite-Padé approximation, isomonodromic deformation and hypergeometric integral, Math. Z. 285 (2017), 397-431, arXiv:1502.06695.

[23] Mazzocco M., Picard and Chazy solutions to the Painlevé VI equation, Math. Ann. 321 (2001), 157-195, math.AG/9901054.

[24] Mironov A., Morozov A., On AGT relation in the case of U(3), Nuclear Phys. B 825 (2010), 1-37, arXiv:0908.2569.

[25] Nekrasov N.A., Seiberg-Witten prepotential from instanton counting, Adv. Theor. Math. Phys. 7 (2003), 831-864, hep-th/0206161.

[26] Okamoto K., Studies on the Painlevé equations. I. Sixth Painlevé equation PVI, Ann. Mat. Pura Appl. 146 (1987), 337-381.

[27] Suzuki T., A particular solution of a Painlevé system in terms of the hypergeometric function ${ }_{n+1} F_{n}, S I G M A$ 6 (2010), 078, 11 pages, arXiv:1002.2685.

[28] Suzuki T., A class of higher order Painlevé systems arising from integrable hierarchies of type A, in Algebraic and geometric aspects of integrable systems and random matrices, Contemp. Math., Vol. 593, Amer. Math. Soc., Providence, RI, 2013, 125-141, arXiv:1002.2685.

[29] Tsuda T., From KP/UC hierarchies to Painlevé equations, Internat. J. Math. 23 (2012), 1250010, 59 pages, arXiv:1004.1347.

[30] Tsuda T., Hypergeometric solution of a certain polynomial Hamiltonian system of isomonodromy type, Q. J. Math. 63 (2012), 489-505, arXiv:1005.4130.

[31] Tsuda T., UC hierarchy and monodromy preserving deformation, J. Reine Angew. Math. 690 (2014), 1-34, arXiv:1007.3450.

[32] Wyllard N., $A_{N-1}$ conformal Toda field theory correlation functions from conformal $\mathcal{N}=2 \mathrm{SU}(N)$ quiver gauge theories, J. High Energy Phys. 2009 (2009), no. 11, 002, 22 pages, arXiv:0907.2189.

[33] Yamada Y., A quantum isomonodromy equation and its application to $\mathcal{N}=2 \mathrm{SU}(N)$ gauge theories, J. Phys. A: Math. Theor. 44 (2011), 055403, 9 pages, arXiv:1011.0292. 